\title{
Schedulability of Bounded-Rate Multi-Mode Systems
}

Rajeev Alur, University of Pennsylvania, Philadelphia, USA

Vojtěch Forejt, Dept. of Computer Science, University of Oxford, UK

Salar Moarref, University of Pennsylvania, Philadelphia, USA

Ashutosh Trivedi, University of Colorado, Boulder, USA

BMMS are hybrid systems that switch freely among a finite set of modes, and whose dynamics is specified by a finite number of real-valued variables with mode-dependent rates that vary within given bounded sets. The scheduler repeatedly proposes a time and a mode while the environment chooses an allowable rate for that mode; the state of the system changes linearly in the direction of the rate. The scheduler aims to keep the state within a safe set, while the environment aimis to leave it. We study the problem of existence of a winning scheduler strategy, and associated complexity questions.

\section{INTRODUCTION}

There is a growing trend towards multi-mode compositional design frameworks $[12 ; 17 ; 13$ for the synthesis of cyber-physical systems where the desired system is built by composing various modes, subsystems, or motion primitives - with well-understood performance characteristics - so as to satisfy certain higher level control objectives. A notable example of such an approach is green scheduling proposed by Nghiem et al. [15, 16 where the goal is to compose different modes of heating, ventilation, and air-conditioning (HVAC) installations in a building so as to keep the temperature surrounding each installation in a given comfort zone while keeping the peak energy consumption under a given budget. Under the assumption that the state of the system grows linearly in each mode, Nghiem et al. gave a polynomial algorithm to decide the green schedulability problem. Alur, Trivedi, and Wojtczak 2 studied general constant-rate multi-mode systems and showed, among others, that the result of Nghiem et al. holds for arbitrary multi-mode systems with constant rate dynamics as long as the scheduler can switch freely among the finite set of modes.

In this paper we present bounded-rate multi-mode systems that generalize constant-rate multi-mode systems by allowing non-constant mode-dependent rates that are given as bounded polytopes. Our motivations to study bounded-rate multi-mode schedulability are twofold. First, it allows one to model a conservative approximation of green schedulability problem in presence of more complex inter-mode dynamics. Second motivation is theoretical and it stems from the desire to characterize decidable problems in context of design and analysis of cyber-physical systems. In particular, we view a bounded-rate multi-mode system as a two-player extension of constant-rate multi-mode system, and show the decidability of schedulability game for such systems.

Before discussing bounded-rate multi-mode system (BMS) in any further detail, let us review the definition, relevant results, and limitations of constant-rate multi-mode system (CMS). A CMS is specified as a finite set of variables whose dynamics in a finite set of modes is given as mode-dependent constant rate vector. The schedulability problem for a CMS and a bounded convex safety set of states is to decide whether there exists an infinite sequence (schedule) of modes and time durations such that choosing modes for corresponding time durations in that sequence keeps the system within the safety set forever. Moreover such schedule is also required to be physically implementable, i.e. the sum of time durations must diverge (the standard non-Zeno requirement [10]). Alur et al. [2 showed that, for the starting states in the interior of the safety set, the necessary and sufficient condition for safe schedulability is the existence of an assignment of dwell times to modes such that the sum of rate vectors of various modes scaled by corresponding dwell time is zero. Intuitively, if it is possible using the modes to loop back to the starting state, i.e. to go to some state other than the starting state and then to return to the starting state, then the same schedule 

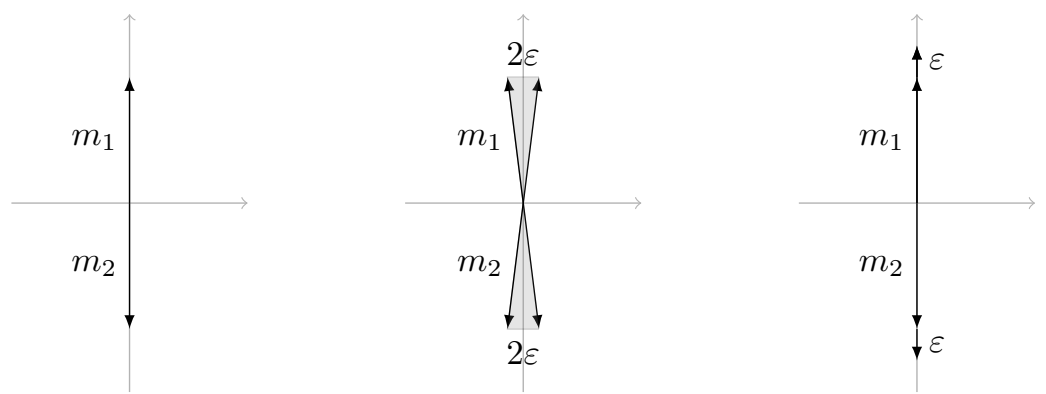

Fig. 1. Multi-mode systems with uncertain rates

can be scaled appropriately and repeated forever to form a periodic schedule that keeps the system inside the interior of any convex safety set while ensuring time divergence. On the other hand, if no such assignment exists then Farkas' lemma implies the existence of a vector such that choosing any mode the system makes a positive progress in the direction of that vector, and hence for any non-Zeno schedule the system will leave any bounded safety set in a finite amount of time. Also, due to constant-rate dynamics such condition can be modeled as a linear program feasibility problem, yielding a polynomial-time algorithm.

Example 1.1. Consider the 2-dimensional CMS shown in Figure 1 (left) with two modes $m_{1}$ and $m_{2}$ with rates of the variables as $\vec{r}_{1}=(0,1)$ in mode $m_{1}$ and $\vec{r}_{2}=(0,-1)$ in mode $m_{2}$. It is easy to see that the system is schedulable for any starting state $\left(x_{0}, y_{0}\right)$ in the interior of any bounded convex set $S$ as $\vec{r}_{1}+\vec{r}_{2}=(0,0)$. The safe schedule consists of the periodic schedule $\left(m_{1}, t\right),\left(m_{2}, t\right)$ for a carefully selected $t \in \mathbb{R}_{>0}$ such that $\left(x_{0}, y_{0}\right)+\vec{r}_{1} t$ stays inside $S$.

However, the schedules constructed in this manner are not robust as an arbitrarily small change in the rate can make the schedule unsafe as shown in the following example.

Example 1.2. Consider a multi-mode system where some environment related fluctuations 10 cause the rate vectors in modes $m_{1}$ and $m_{2}$ to differ from those in Example 1.1 by an arbitrarily small $\varepsilon>0$ as shown in Figure 1 (center). Here, $m_{1}$ can have rate-vectors from $\{(0+\delta, 1):-\varepsilon \leq \delta \leq \varepsilon\}$, while rate-vectors of $m_{2}$ are from $\{(0+\delta,-1):-\varepsilon \leq \delta \leq \varepsilon\}$. First we show that the periodic schedule $\left(m_{1}, t\right),\left(m_{2}, t\right)$ proposed in Example 1.1 is not safe for any $t$. Consider the case when the rate vector in modes $m_{1}$ and $m_{2}$ are fixed to $(\varepsilon, 1)$ and $(\varepsilon,-1)$. Starting from the state $\left(x_{0}, y_{0}\right)$ and following the periodic schedule $\left(m_{1}, t\right),\left(m_{2}, t\right)$ for $k$ steps the state of the system will be $\left(x_{0}+k t \varepsilon, y_{0}\right)$ after $k$ steps. Hence it is easy to see that for any bounded safety set the state of the system will leave the safety set after finitely many steps. In fact, for this choice of rate vectors no non-Zeno safe schedule exists at all, since by choosing any mode for a positive time the system makes a positive progress along the $X$ axis.

We formalize modeling of such multi-mode system under uncertainty as bounded-rate multimode systems (BMS). BMSs can also approximate 7 the effect of more complex non-linear, and even time-varying, mode dynamics over a bounded safety set. Formally, a BMS is specified as a finite set of variables whose dynamics in a finite set of modes is given as a mode-dependent bounded convex polytopes of rate vectors. We present the schedulability problem on BMS as an infinite-round zero-sum game between two players, the scheduler and the environment; at each round the scheduler chooses a mode and a time duration, the environment chooses a rate vector from the allowable set of rates for that mode, and the state of the system is evolved accordingly. The recipe for selecting their choices, or moves, is formalized in the form of a strategy that is a function of the history of the game so far to 
a move of the player. A strategy is called positional if it is a function of the current state. We say that the scheduler wins the schedulability game, or has a winning strategy, from a given starting state if there is a scheduler strategy such that, irrespective of the strategies of the environment, the state of the system stays within the safety set and time does not converge to any real number. Similarly, we say that the environment has a winning strategy if she has a strategy such that for any strategy of the scheduler the system leaves the safety set in a finite amount of time, or the time converges to some real number. One of the central results of this paper is that the schedulability games on BMS are determined, i.e. for each starting state exactly one of the players has a winning strategy. Note that the determinacy of these games could be proved using more general results on determinacy, e.g. [14], however our proof is direct and shows the existence of positional winning strategies.

We distinguish between two kind of strategies of the scheduler-the static strategies, where the scheduler can not observe the decisions of the environment, and the dynamic strategies, where the scheduler can observe the decisions of the environment so far before choosing a mode and a time. Our use of static vs. dynamic strategies closely corresponds to standard open-loop control vs. closed-loop control distinction in control theory. Also notice that static strategies correspond precisely to schedules, and we often use these two terms interchangeably. A key challenge in the schedulability analysis of BMS is inadequacy of static strategies as shown below.

Example 1.3. Consider the BMS of Figure1 (right) where the rates in mode $m_{1}$ and $m_{2}$ lie in $\{(0,1+\delta): 0 \leq \delta \leq \varepsilon\}$ and $\{(0,-(1+\delta)): 0 \leq \delta \leq \varepsilon\}$, respectively. We hint that there is no static winning strategy of the scheduler in this BMS (the formal conditions for the existence of static winning strategies will be analyzed later in the paper). Let us assume, for example, that $\sigma=\left(m_{1}, t_{1}\right),\left(m_{2}, t_{2}\right), \ldots$ is a static non-Zeno winning strategy of the scheduler. Moreover consider two strategies $\pi$ and $\pi^{\prime}$ of the environment that differ only in mode $m_{1}$ where they propose rates $(1,0)$ and $(1+\varepsilon, 0)$ respectively. Let $\varrho$ and $\varrho^{\prime}$ be the sequences of system states and player's choices - what we subsequently refer to as runs - as the game progresses from a starting state $\left(x_{0}, y_{0}\right)$ where the environment uses the strategy $\pi$ and $\pi^{\prime}$, respectively, against the scheduler's strategy $\sigma$. Let $T_{1}(i)$ and $T_{2}(i)$ be the time spent in mode $m_{1}$ and $m_{2}$, resp., till the $i$-th round in runs $\varrho$ and $\varrho^{\prime}$, while $T_{1}$ and $T_{2}$ be total time spent in mode $m_{1}$ and $m_{2}$, resp. The state of the system in the runs $\varrho$ and $\varrho^{\prime}$ after $i$ rounds will be $\left(x_{0}, y_{0}+T_{1}(i)-T_{2}(i)\right)$ and $\left(x_{0}, y_{0}+T_{1}(i)-T_{2}(i)+T_{1}(i) \varepsilon\right)$. Hence the distance $T_{1}(i) \varepsilon$ between states reached after $i$-rounds in runs $\varrho$ and $\varrho^{\prime}$ tends to $T_{1} \varepsilon$ as $i$ tends to $\infty$. It is easy to see that if $\sigma$ is a winning strategy then $T_{1}=\infty$; since if $T_{1}<\infty$ and $T_{2}=\infty$ then the system will move in the direction of rates of mode $m_{2}$, while if both $T_{1}$ and $T_{2}$ are finite then the strategy is not non-Zeno. Hence the system will eventually leave any bounded safety set, contradicting our assumption on $\sigma$ being a winning strategy.

The techniques used for schedulability analysis and schedule construction for CMS cannot be generalized to BMS since in a BMS, the scheduler may not have a strategy to loop back to the starting state. In fact, in general the scheduler does not have a strategy to revisit any state as is clear from Figure 1 (right) - here the environment can always choose a rate vector in both mode $m_{1}$ and $m_{2}$ to avoid any previously visited state. However, from our results on BMS it follows that if the scheduler has a winning strategy then he has a strategy to restrict the future states of the system to a ball of arbitrary diameter centered around the starting state.

In order to solve schedulability game for BMS we exploit the following observation ${ }^{1}$ the scheduler has a winning strategy, from all the starting states in the interior of the safety set $S$, if and only if there is a polytope $P \subseteq S$, such that for every vertex $\bar{v}$ of $P$ there is a mode

${ }^{1}$ Blanchini [4. Theorem 5.2] makes a similar observation to design uniformly stable control for uncertain linear systems. 
$m(\bar{v})$ and time $t(\bar{v})$ such that choosing mode $m(\bar{v})$ for time $t(\bar{v})$ from the vertex $\bar{v}$, the line $\bar{v}+\vec{r} t(\bar{v})$ stays within polytope $P$ for all allowable rates $\vec{r}$ of $m(\bar{v})$. In other words, for any vertex of $P$ there is a mode and a time duration such that if the system evolves with any rate vector of that mode for such amount of time, the system stays in $P$. For a BMS $\mathcal{H}$ we call such a polytope $\mathcal{H}$-closed. We show how such a polytope can be constructed for a BMS based on its characteristics. We also analyze the complexity of such a construction. The existence of an $\mathcal{H}$-closed polytope immediately provides a non-Zeno safe dynamic strategy for the scheduler for any starting state in $P$ : find the convex coefficient $\left(\lambda_{1}, \lambda_{2}, \ldots, \lambda_{k}\right)$ of the current state $\bar{x}$ with respect to the finite set of vertices $\left(\bar{x}_{1}, \bar{x}_{2}, \ldots, \bar{x}_{k}\right)$ of $P$ and choose the mode $m\left(\bar{x}_{i}\right)$ for time $t\left(\bar{x}_{i}\right) \lambda_{i}$ that maximizes $t\left(\bar{x}_{i}\right) \lambda_{i}$. Then, for some choice $\vec{r}$ of the environment for $m\left(\bar{x}_{i}\right)$ the system will progress to $\bar{x}^{\prime}=\bar{x}+t\left(\bar{x}_{i}\right) \lambda_{i} \vec{r}$. One can repeat this dynamic strategy from the next state $\bar{x}^{\prime}$ as the current state. We prove that such strategy is both non-Zeno and safe.

An extreme-rate CMS of a BMS $\mathcal{H}$ is obtained by preserving the set of modes, and for each mode assigning a rate which is a vertex of the available rate-set of that mode. The main result of the paper is that an $\mathcal{H}$-closed polytope exists for a BMS $\mathcal{H}$ iff all extremerate CMSs of $\mathcal{H}$ are schedulable. The "only if" direction of the above characterization is immediate as if some extreme-rate CMS is not schedulable then the environment can fix those rate vectors and win the schedulability game in the BMS. We show the "if" direction by explicitly constructing the $\mathcal{H}$-closed polytope.

Example 1.4. Consider the BMS $\mathcal{H}$ from Figure 1 (right) with $\varepsilon=0.5$. The safety set is given as a shaded area in Figure 2 (left) and $\bar{x}_{0}=(-1,-0.5)$ is the initial state. Observe that all extreme-rate combinations are schedulable and hence we show a winning strategy. An $\mathcal{H}$-closed polytope for this BMS is the line-segment between the points $(0,2.5)$ and $(0,-2.5)$ (we explain the construction of such a polytope in Section 3). After translating this line-segment to $x_{0}$ and scaling it to fit inside the safety set, we will get the line-segment connecting $\bar{x}_{1}=(-1,1)$ to $\bar{x}_{2}=(-1,-2)$, as shown in Figure 2 (left). At vertices $\bar{x}_{1}$ and $\bar{x}_{2}$ modes $m_{2}$ and $m_{1}$, respectively, can be used for 1 time unit. A winning strategy of the scheduler is to keep the system's state along the line segment. Our strategy observes the current state $\bar{x}$ and finds the mode to choose by computing convex coefficient $\lambda \in[0,1]$ such that $\bar{x}=\lambda \bar{x}_{1}+(1-\lambda) \bar{x}_{2}$. For instance, at state $\bar{x}_{0}=\frac{1}{2} \bar{x}_{1}+\frac{1}{2} \bar{x}_{2}$ the scheduler can choose any of the modes for $\frac{1}{2}$ time units. Assume that it chooses $m_{1}$. Based on environment's choice the state of system after $\frac{1}{2}$ time units will be in the set $\{-1,0.5+\delta: 0 \leq \delta \leq 0.5\}$. The scheduler observes this new state after $\frac{1}{2}$ time-unit, and chooses mode and time accordingly. For example, if the environment chooses $(0,1.25)$ and so the next state is $\bar{x}=(-1,0.75)=$ $\frac{1}{12} \bar{x}_{1}+\frac{11}{12} \bar{x}_{2}$, the scheduler can choose mode $m_{2}$ for $\frac{11}{12}$ time units. In Figure 2 (right) we show first two rounds of the game. Since, for any point on our line segment the scheduler can choose a mode for at least 0.5 time unit and stay on the line segment, such strategy is both safe and non-Zeno.

We also extend the above result to decide the winner starting from arbitrary states, i.e. including those states that lie on the boundary of the safety set. Here we show that the existence of a winning scheduler strategy implies the existence of a winning scheduler strategy which only allows to move from lower-dimensional faces to higher-dimensional ones and not the other way around; this allows us to use an algorithm which traverses the face lattice of the safety set and analyses each face one by one. We also prove co-NP completeness of the schedulability problem, showing the hardness by giving a reduction from 3-SAT to the non-schedulability problem. On a positive note, we show that if the number of variables is fixed, then the schedulability game can be decided in polynomial time. This is because in such a case we can prove that there is only polynomially many candidates for falsifiers we need to consider, and hence we can check each of them one by one. Our proof technique 

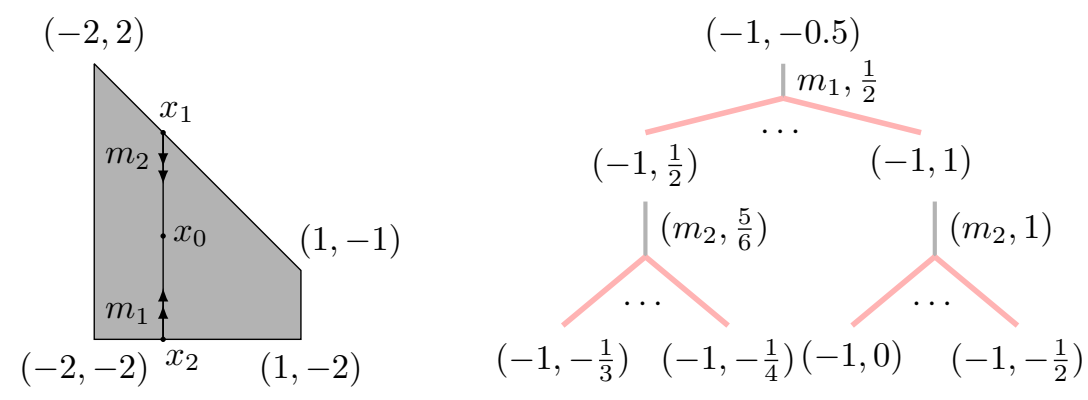

Fig. 2. $\mathcal{H}$-closed polytope and dynamic strategy

follows the same direction as a proof for fixed-parameter complexity in 3 . However, the result of [3] only applies to the case where the scheduler aims to stay within $\varepsilon$ distance of the starting point for some $\varepsilon>0$; our result applies also to cases where the starting point is on the boundary of the safety set, and so just keeping within a small distance of the starting point would not be sufficient. Finally, we study a discrete version of schedulability games where the scheduler can choose time delays only at multiples of a given clock period, while the environment can choose rate vectors from a finite set. We show that discrete schedulability games on BMS are EXPTIME-complete, and that the maximal clock period for which the scheduler has a winning strategy can be computed in exponential time. If the system is a CMS, we get a PSPACE algorithm, improving the result of [2] where only an approximation of the maximal clock period for CMS was studied.

For a review of related work on CMS and green scheduling we refer to 16,15 and 2 . The work closest to this paper is that on constrained regulator problem by Blanchini 4 . This work studies the concept of positively invariant region that closely corresponds to our definition of $\mathcal{H}$-closed polytope, and present conditions for schedulability of more general linear systems. However, [4 does not settle computational complexity of corresponding problem that form the core of our results. We refer the reader to [5] for an excellent survey on positively invariant sets and their applications in controller synthesis. Another closely related work is by Heymann et al. 10 that considers scheduling problem on BMS where rate-vectors are given as upper and lower rate matrices and the safety set as the entire nonnegative orthant. The main result of [10] is that the scheduler wins if he wins in the CMS of the lower rate matrix, and wins only if he wins in the CMS of the upper rate matrix. We study more general BMS and safety sets, and characterize necessary and sufficient condition for schedulability. Finally, to complete the picture, we remark that games on hybrid automata 9,8 , that correspond to BMS with local invariants and guards, have undecidable schedulability problem.

This paper is based on a conference publication 1. Compared to the previous version where the polynomial-time algorithm was given only for two dimensions, this paper provides a polynomial-time algorithm for any fixed number of dimensions. Also, this paper contains full proofs of all results presented.

\section{PROBLEM DEFINITION}

Points and Vectors. Let $\mathbb{R}$ be the set of real numbers, and let $\mathbb{R}_{>0}$ be the set of nonnegative real numbers. We represent the states in our system as points in $\mathbb{R}^{n}$, and we equip this space with the standard Euclidean norm $\|\cdot\|$. We denote points in this state space by $\bar{x}, \bar{y}$, vectors by $\vec{r}, \vec{v}$, and the $i$-th coordinate of point $\bar{x}$ and vector $\vec{r}$ by $\bar{x}(i)$ and $\vec{r}(i)$, respectively. We write $\overrightarrow{0}$ for a vector with all its coordinates equal to 0 ; its dimension is often clear from the context. The distance $\|\bar{x}, \bar{y}\|$ between points $\bar{x}$ and $\bar{y}$ is defined as 
$\|\bar{x}-\bar{y}\|$. For two vectors $\vec{v}_{1}, \vec{v}_{2} \in \mathbb{R}^{n}$, we write $\vec{v}_{1} \cdot \vec{v}_{2}$ to denote their dot product defined as $\sum_{i=1}^{n} \vec{v}_{1}(i) \cdot \vec{v}_{2}(i)$. Given vectors $\vec{v}_{1}, \ldots \vec{v}_{n}$, by $\operatorname{span}\left(\vec{v}_{1}, \ldots, \vec{v}_{n}\right)$ we denote the smallest vector space containing $\vec{v}_{1}, \ldots, \vec{v}_{n}$. In particular, for an empty sequence this is the empty space. We sometimes also use $\operatorname{span}\left(\vec{v}_{1}, \ldots, \vec{v}_{n}\right)$ to refer to the set of vectors of the space. For a vector space $V$, we use $\operatorname{dim}(V)$ for the dimension of the space, i.e. the size of the linear basis of $V$. We sometimes use the shorthand $\operatorname{dim}\left(\vec{v}_{1}, \ldots, \vec{v}_{n}\right)$ instead of $\operatorname{dim}\left(\operatorname{span}\left(\vec{v}_{1}, \ldots, \vec{v}_{n}\right)\right)$.

Boundedness and Interior.. We denote a closed ball of radius $d \in \mathbb{R}_{\geq 0}$ centered at $\bar{x}$ as $B_{d}(\bar{x})=\left\{\bar{y} \in \mathbb{R}^{n}:\|\bar{x}, \bar{y}\| \leq d\right\}$. We say that a set $S \subseteq \mathbb{R}^{n}$ is bounded if there exists $d \in \mathbb{R}_{\geq 0}$ such that for all $\bar{x}, \bar{y} \in S$ we have $\|\bar{x}, \bar{y}\| \leq d$. The interior of a set $S, \operatorname{int}(S)$, is the set of all points $\bar{x} \in S$ for which there exists $d>0$ such that $B_{d}(\bar{x}) \subseteq S$.

Convexity.. A point $\bar{x}$ is a convex combination of a finite set of points $X=\left\{\bar{x}_{1}, \bar{x}_{2}, \ldots, \bar{x}_{k}\right\}$ if there are $\lambda_{1}, \lambda_{2}, \ldots, \lambda_{k} \in[0,1]$ such that $\sum_{i=1}^{k} \lambda_{i}=1$ and $\bar{x}=\sum_{i=1}^{k} \lambda_{i} \cdot \bar{x}_{i}$. The convex hull of $X$ is then the set of all points that are convex combinations of points in $X$. We say that $S \subseteq \mathbb{R}^{n}$ is convex iff for all $\bar{x}, \bar{y} \in S$ and all $\lambda \in[0,1]$ we have $\lambda \bar{x}+(1-\lambda) \bar{y} \in S$ and moreover, $S$ is a convex polytope if it is bounded and there exists $k \in \mathbb{N}$, a matrix $A$ of size $k \times n$ and a vector $\vec{b} \in \mathbb{R}^{k}$ such that $\bar{x} \in S$ iff $A \bar{x} \leq \vec{b}$. We write rows $(M)$ for the number of rows in a matrix $M$, here $\operatorname{rows}(A)=k$.

A point $\bar{x}$ is a vertex of a convex polytope $P$ if it is not a convex combination of two distinct (other than $\bar{x}$ ) points in $P$. For a convex polytope $P$ we write vert $(P)$ for the finite set of points that correspond to the vertices of $P$. Each point in $P$ can be written as a convex combination of the points in vert $(P)$, or in other words, $P$ is the convex hull of $\operatorname{vert}(P)$. From standard properties of polytopes, it follows that for every convex polytope $P$ and every vertex $\bar{c}$ of $P$, there exists a vector $\vec{v}$ such that $\vec{v} \cdot \bar{c}=d$ and $\vec{v} \cdot \bar{x}>d$ for all $\bar{x} \in P \backslash\{\bar{c}\}$ for some $d$. We call such a vector $\vec{v}$ a supporting hyperplane of the polytope $P$ at $\bar{c}$.

\subsection{Multi-Mode Systems}

A multi-mode system is a hybrid system equipped with finitely many modes and finitely many real-valued variables. A configuration is described by values of the variables, which change, as the time elapses, at the rates determined by the modes being used. The choice of rates is nondeterministic, which introduces a notion of adversarial behavior. Formally,

Definition 2.1 (Multi-Mode Systems). A multi-mode system is a tuple $\mathcal{H}=(M, n, \mathcal{R})$ where: $M$ is the finite nonempty set of modes, $n$ is the number of continuous variables, and $\mathcal{R}: M \rightarrow 2^{\mathbb{R}^{n}}$ is the rate-set function that, for each mode $m \in M$, gives a set of vectors.

We often write $\vec{r} \in m$ for $\vec{r} \in \mathcal{R}(m)$ when $\mathcal{R}$ is clear from the context. A finite run of a multi-mode system $\mathcal{H}$ is a finite sequence of states, timed moves and rate vector choices $\varrho=\left\langle\bar{x}_{0},\left(m_{1}, t_{1}\right), \vec{r}_{1}, \bar{x}_{1}, \ldots,\left(m_{k}, t_{k}\right), \vec{r}_{k}, \bar{x}_{k}\right\rangle$ such that for all $1 \leq i \leq k$ we have $\vec{r}_{i} \in \mathcal{R}\left(m_{i}\right)$ and $\bar{x}_{i}=\bar{x}_{i-1}+t_{i} \cdot \vec{r}_{i}$. For such a run $\varrho$ we say that $\bar{x}_{0}$ is the starting state, while $\bar{x}_{k}$ is its last state. An infinite run is defined in a similar manner. We write Runs and FRuns for the set of infinite and finite runs of $\mathcal{H}$, while $\operatorname{Runs}(\bar{x})$ and $F R u n s(\bar{x})$ for the set of infinite and finite runs starting from $\bar{x}$.

An infinite run $\left\langle\bar{x}_{0},\left(m_{1}, t_{1}\right), \vec{r}_{1}, \bar{x}_{1},\left(m_{2}, t_{2}\right), \vec{r}_{2}, \ldots\right\rangle$ is Zeno if $\sum_{i=1}^{\infty} t_{i}<\infty$. Given a set $S \subseteq \mathbb{R}^{n}$ of safe states, we say that a run $\left\langle\bar{x}_{0},\left(m_{1}, t_{1}\right), \vec{r}_{1}, \bar{x}_{1},\left(m_{2}, t_{2}\right), \vec{r}_{2}, \ldots\right\rangle$ is $S$-safe if for all $i \geq 0$ we have that $\bar{x}_{i} \in S$ and $\bar{x}_{i}+t \cdot \vec{r}_{i+1} \in S$ for all $t \in\left[0, t_{i+1}\right]$. Notice that if $S$ is a convex set and $\bar{x}_{i} \in S$ for all $i \geq 0$, then for all $i \geq 0$ and for all $t \in\left[0, t_{i+1}\right]$ we have that $\bar{x}_{i}+t \cdot \vec{r}_{i+1} \in S$. The concept of $S$-safety for finite runs is defined in a similar manner. Sometimes we simply call a run safe when the safety set and the starting state are clear from the context. 
We formally give the semantics of a multi-mode system $\mathcal{H}$ as a turn-based two-player game between the players, the scheduler and the environment, who choose their moves to construct a run of the system. The system starts in a given starting state $\bar{x}_{0} \in \mathbb{R}^{n}$ and at each turn the scheduler chooses a timed move, a pair $(m, t) \in M \times \mathbb{R}_{>0}$ consisting of a mode and a time duration, and the environment chooses a rate vector $\vec{r} \in \mathcal{R}(m)$ and as a result the system changes its state from $\bar{x}_{0}$ to the state $\bar{x}_{1}=\bar{x}_{0}+t \cdot \vec{r}$ in $t$ time units following the linear trajectory according to the rate vector $\vec{r}$. From the next state $\bar{x}_{1}$ the scheduler again chooses a timed move and the environment an allowable rate vector, and the game continues forever in this fashion.

Given a bounded and convex safety set $S$, we define (safe) schedulability objective $\mathcal{W}_{\text {Safe }}^{S}$ as the set of $S$-safe and non-Zeno runs of $\mathcal{H}$. In a schedulability game the winning objective of the scheduler is to make sure that the constructed run of a system belongs to $\mathcal{W}_{\text {Safe }}^{S}$, while the goal of the environment is the opposite. The selection mechanism of the players is typically defined as strategies. A strategy $\sigma$ of the scheduler is function $\sigma: F R u n s \rightarrow M \times \mathbb{R}_{\geq 0}$ that gives a timed move for every history of the game. A strategy $\pi$ of the environment is a function $\pi:$ FRuns $\times\left(M \times \mathbb{R}_{\geq 0}\right) \rightarrow \mathbb{R}^{n}$ that chooses an allowable rate for a given history of the game and choice of the scheduler. We say that a strategy is positional if it suggests the same action for all runs with the common last state. We write $\Sigma$ and $\Pi$ for the set of strategies of the scheduler and the environment, respectively.

Given a starting state $\bar{x}_{0}$ and a strategy pair $(\sigma, \pi) \in \Sigma \times \Pi$ we define the unique run $\operatorname{Run}\left(\bar{x}_{0}, \sigma, \pi\right)$ starting from $\bar{x}_{0}$ as

$$
\operatorname{Run}\left(\bar{x}_{0}, \sigma, \pi\right)=\left\langle\bar{x}_{0},\left(m_{1}, t_{1}\right), \vec{r}_{1}, \bar{x}_{1},\left(m_{2}, t_{2}\right), \vec{r}_{2}, \ldots\right\rangle
$$

where for all $i \geq 1$ we have that $\left(m_{i}, t_{i}\right)=\sigma\left(\left\langle\bar{x}_{0},\left(m_{1}, t_{1}\right), \vec{r}_{1}, \bar{x}_{1}, \ldots, \bar{x}_{i-1}\right\rangle\right)$ and $\vec{r}_{i}=$ $\pi\left(\left\langle\bar{x}_{0},\left(m_{1}, t_{1}\right), \vec{r}_{1}, \bar{x}_{1}, \ldots, \bar{x}_{i-1},\left(m_{i}, t_{i}\right)\right\rangle\right)$ and $\bar{x}_{i}=\bar{x}_{i-1}+t_{i} \cdot \vec{r}_{i}$. The scheduler wins the game if there is $\sigma \in \Sigma$ such that for all $\pi \in \Pi$ we get $\operatorname{Run}\left(\bar{x}_{0}, \sigma, \pi\right) \in \mathcal{W}_{\text {Safe }}^{S}$. Such a strategy $\sigma$ is winning. Similarly, the environment wins the game if there is $\pi \in \Pi$ such that for all $\sigma \in \Sigma$ we have $\operatorname{Run}\left(\bar{x}_{0}, \sigma, \pi\right) \notin \mathcal{W}_{\text {Safe }}^{S}$. Again, $\pi$ is called winning in this case. If a winning strategy for the scheduler exists, we say that $\mathcal{H}$ is schedulable for $S$ and $\bar{x}_{0}$ (or simply schedulable if $S$ and $\bar{x}_{0}$ are clear from the context). The following is the main algorithmic problem studied in this paper.

Definition 2.2 (Schedulability). Given a multi-mode system $\mathcal{H}$, a safety set $S$, and a starting state $\bar{x}_{0} \in S$, the (safe) schedulability problem is to decide whether there exists a winning strategy of the scheduler.

\subsection{Bounded-Rate Multi-Mode Systems}

To algorithmically decide the schedulability problem, we need to restrict the range of $\mathcal{R}$ and the domain of safety set $S$ in a schedulability game on a multi-mode system. The most general model that we consider are the bounded-rate multi-mode systems (BMS) that are multi-mode systems $(M, n, \mathcal{R})$ such that $\mathcal{R}(m)$ is a convex polytope for every $m \in M$. We also assume that the safety set $S$ is specified as a convex polytope. In our proofs we often refer to another variant of multi-mode systems in which there are only a fixed number of different rates in each mode (i.e. $\mathcal{R}(m)$ is finite for all $m \in M$ ). We call such a multimode system multi-rate multi-mode systems (MMS). Finally, a special form of MMS are constant-rate multi-mode systems (CMS) 2 in which $\mathcal{R}(m)$ is a singleton for all $m \in M$. We sometimes use $\mathcal{R}(m)$ to refer to the unique element of the set $\mathcal{R}(m)$ in a CMS. The concepts for the schedulability games for BMS and MMS are already defined for multimode systems. Similar concepts also hold for CMS but note that the environment has no real choice in this case. For this reason, we can refer to a schedulability game on CMS as a one-player game. 


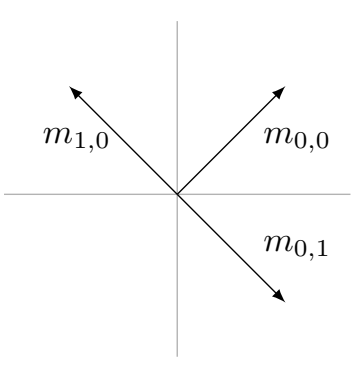

(a) Constant-Rate

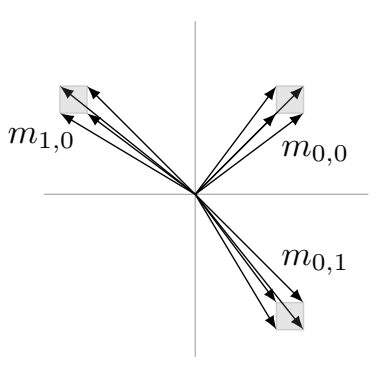

(b) Bounded-Rate

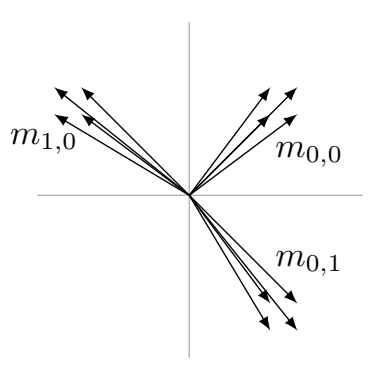

(c) Multi-Rate

Fig. 3. Restricted Multi-mode Systems

A key 2 practical motivation for studying CMS was to generalize results on green scheduling problem by Nghiem et al. [16]. We argue that BMS are a suitable abstraction to study green scheduling problem when various rates of temperature change are either uncertain or follow a complex and time-varying dynamics, as shown in the following example.

Example 2.3 (Green Scheduling). Consider a building with two rooms $A$ and B. HVAC units in each zone can be in one of the two modes $0(\mathrm{OFF})$ and $1(\mathrm{ON})$. We write the mode of the combined system as $m_{i, j}$ to represent the fact that rooms $A$ and $B$ are in mode $i \in\{0,1\}$ and $j \in\{0,1\}$, respectively. The rate of temperature change and the energy usage for each room is given below.

\begin{tabular}{|l|l|l|}
\hline Zones & ON & OFF \\
\hline A (temp. change rate/ usage) & $-2 / 2$ & $2 / 1$ \\
\hline B (temp. change/ usage) & $-2 / 2$ & $2 / 1$ \\
\hline
\end{tabular}

Following [2] we assume that the energy cost is equal to energy usage if the peak energy usage at any given point in time is less than or equal to 3 units, otherwise the energy cost is 10 times of that standard rate. It follows that to minimize energy cost the peak usage, if possible, must not be higher than 3 units at any given time. We can model the system as a CMS with modes $m_{0,0}, m_{0,1}$, and $m_{1,0}$, because these are the only ones that have the peak usage at most 3 . The variables of the CMS are the temperature of the rooms, while the safety set is the constraint that temperature of both zones should be between $65^{\circ} \mathrm{F}$ to $75^{\circ} \mathrm{F}$. The existence of a winning strategy in CMS implies the existence of a controller for the HVAC unit with energy peak demand less than or equal to 3 units. In Figure 3 (a) we show a graphical representation of such CMS with three modes $m_{0,0}, m_{0,1}$ and $m_{1,0}$ and two variables (corresponding to the two axes). The rate of the variables in mode $m_{0,0}$ is $(2,2)$, in mode $m_{0,1}$ is $(2,-2)$, and in mode $m_{1,0}$ is $(-2,2)$.

Now assume that the rate of temperature change in a mode is not constant and can vary within a given margin $\varepsilon>0$. Schedulability problem for such system can best be modeled as a BMS as shown in Figure 3. (b) where the polytope of possible rate vectors is shown as a shaded region. In Figure 3 . (c) we show a MMS where variables can only change with the extreme rates of the BMS in Figure 3 .(b).

We say that a CMS $H=(M, n, R)$ is an instance of a multi-mode system $\mathcal{H}=(M, n, \mathcal{R})$ if for every $m \in M$ we have that $R(m) \in \mathcal{R}(m)$. For example, the CMS shown in Figure 3 (a) is an instance of BMS in Figure 3.(b). We denote the set of instances of a multi-mode system $\mathcal{H}$ by $\llbracket \mathcal{H} \rrbracket$. Notice that for a BMS $\mathcal{H}$ the set $\llbracket \mathcal{H} \rrbracket$ of its instances is uncountably infinite, while for a MMS $\mathcal{H}$ the set $\llbracket \mathcal{H} \rrbracket$ is finite, and of size exponential in the size of $\mathcal{H}$. We say that a $\operatorname{MMS}\left(M, n, \mathcal{R}^{\prime}\right)$ is the extreme-rate $\operatorname{MMS}$ of a $\operatorname{BMS}(M, n, \mathcal{R})$ if $\mathcal{R}^{\prime}(m)=\operatorname{vert}(\mathcal{R}(m))$. 
The MMS in Figure 3.(c) is the extreme-rate MMS for the BMS in Figure 3.(b). We write $\operatorname{Ext}(\mathcal{H})$ for the extreme-rate MMS of the BMS $\mathcal{H}$.

Notice that for every starting state and winning objective at most one player can have a winning strategy. We say that a game is not determined if no player has a winning strategy for some starting state.

\section{SOLVING SCHEDULABILITY GAMES}

In this section we discuss the decidability of the schedulability problem for BMS. We first present a solution for the case when the starting state is in the interior of a safety set, and then generalize it to arbitrary starting states in Section 3.2

\subsection{Starting State in the Interior of Safety Set}

Alur et al. 2 presented a polynomial-time algorithm to decide if the scheduler has a winning strategy in a schedulability game on a CMS for an arbitrary starting state. In particular, for starting states in the interior of the safety set, they characterized a necessary and sufficient condition.

Theorem $3.1(\sqrt{2})$. The scheduler has a winning strategy in a CMS $H=(M, n, R)$, with convex safety set $S$ and starting state $\bar{x}_{0}$ in the interior of $S$, iff there is $\vec{t} \in \mathbb{R}_{\geq 0}^{|M|}$ satisfying:

$$
\sum_{i=1}^{|M|} R(i)(j) \cdot \vec{t}(i)=0 \text { for } 1 \leq j \leq n \text { and } \sum_{i=1}^{|M|} \vec{t}(i)=1 .
$$

We call a CMS safe if it satisfies (1) and we call $H$ unsafe otherwise. The intuition behind Theorem 3.1 is that the scheduler has a winning strategy if and only if it is possible to return to the starting state in strictly positive time units. From the results of 22 it also follows that whenever a winning strategy exists, there is a strategy which does not look at a history or even the current state, but only uses a bounded counter of size $\ell \leq|M|-1$ and after a history of length $k$ makes a decision only based on the number $k$ modulo $\ell$. Such strategies are called periodic.

It is natural to ask whether the approach of 2 can be generalized to BMS. Unfortunately, Example 1.3 shows that in a BMS, although a winning strategy may exist, it may not be possible to return to the initial state, or indeed visit any state twice. Another natural question to ask is whether a suitable generalization of periodic strategies suffice for BMS. Static strategies are BMS analog of periodic strategies that behave in the same manner irrespective of the choices of the environment, i.e. for a static strategy $\sigma$ we have that $\sigma(\rho)=\sigma\left(\rho^{\prime}\right)$ for all runs $\rho=\left\langle\bar{x}_{0},\left(m_{1}, t_{1}\right), \vec{r}_{1}, \bar{x}_{1}, \ldots,\left(m_{k}, t_{k}\right), \vec{r}_{k}, \bar{x}_{k}\right\rangle$ and $\rho^{\prime}=\left\langle\bar{x}_{0},\left(m_{1}, t_{1}\right), \vec{r}_{1}^{\prime}, \bar{x}_{1}^{\prime}, \ldots,\left(m_{k}, t_{k}\right), \vec{r}_{k}^{\prime}, \bar{x}_{k}^{\prime}\right\rangle$. Static strategies are often desirable in the settings where the scheduler can not observe the state of the system. However, we observe in the following proposition that except for the degenerate cases when the BMS contains a subset of modes which induce a safe CMS, the scheduler can never win a game on BMS using static strategies. We saw an example of this phenomenon in Figure 1.(c).

Proposition 3.2. For a given starting state in the interior of the safety set $S$, the scheduler has a static winning strategy in a $B M S \mathcal{H}=(M, n, \mathcal{R})$ iff there is $M^{\prime} \subseteq M$ such that $|\mathcal{R}(m)|=1$ for all $m \in M^{\prime}$, and the CMS $H=\left(M^{\prime}, n, R\right)$ is safe, where $R(m)$ is the unique rate of $\mathcal{R}(m)$.

Proof. The "if" direction is trivial. To show the "only if" direction we show that if there is no CMS subsystem of $\mathcal{H}$ for which there is a safe and non-Zeno schedule, then there is no static winning schedule for schedulability objective. Let $\sigma=\left(m_{1}, t_{1}\right),\left(m_{2}, t_{2}\right), \ldots$ be a static schedule. 
Assume there is $m \in M$ with two different rates $\vec{r}_{a}$ and $\vec{r}_{b}$ such that $\sum_{i: m_{i}=m} t_{i}=\infty$. We then define two strategies for the environment, $\pi_{a}$ and $\pi_{b}$ which for a mode $m$ always pick a rate $\vec{r}_{a}$ and $\vec{r}_{b}$, respectively. After the first $k$ steps, the point reached under $\sigma_{b}$ is equal to

$$
\bar{x}_{b}=\bar{x}_{a}+\left(\vec{r}_{b}-\vec{r}_{a}\right) \cdot \sum_{i \leq k: m_{i}=m} t_{i}
$$

Hence, the points $\bar{x}_{a}$ and $\bar{x}_{b}$ will be arbitrarily far apart for large enough $k$, since the safety set is bounded, one of the strategies $\pi_{a}$ and $\pi_{b}$ must ensure that a point outside is left eventually.

On the other hand, assume all modes $m$ which have two different rates satisfy that $\sum_{i: m_{i}=m} t_{i}$ is finite. Let $\bar{M}$ be all such modes, and let $d_{1}:=\|\vec{r}\| \cdot \sum_{i: m_{i} \in \bar{M}} t_{i} \leq \infty$ where $\vec{r}$ is the rate with the maximal Euclidean norm which occurs in $\mathcal{H}$. Intuitively, $d$ is the upper bound on the change of the values of variables caused by using the modes of $\bar{M}$. Let $d_{2}$ be the diametre of the safety set $S$, and let $\gamma$ be the Euclidean distance of the initial point $\bar{x}_{0}$ from the boundary of $S$. We define a strategy

$$
\sigma^{\prime}=\left(m_{1}^{\prime}, t_{1}^{\prime} \cdot \frac{\gamma}{d_{1}+d_{2}}\right),\left(m_{2}^{\prime}, t_{2}^{\prime} \frac{\gamma}{d_{1}+d_{2}}\right), \ldots
$$

where $\left(m_{1}^{\prime}, t_{1}^{\prime}\right),\left(m_{2}^{\prime}, t_{2}\right), \ldots$ is the sequence $\left(m_{1}, t_{1}\right),\left(m_{2}, t_{2}\right) \ldots$ from which we omit all the tuples which have a mode from $\bar{M}$ in the first component. The strategy $\sigma^{\prime}$ is safe, because for any sequence of rates $\vec{r}_{1}, \vec{r}_{2} \ldots$ with $\vec{r}_{i} \in m_{i}$, and for every $k$ we have:

$$
\begin{aligned}
\left.\frac{\gamma}{d_{1}+d_{2}} \cdot \| \sum_{i \leq k} \vec{r}_{i} \cdot t_{i}-\sum_{i \leq k, m_{i} \in \bar{M}} \vec{r}_{i} \cdot t_{i}\right) \| & \left.\leq \frac{\gamma}{d_{1}+d_{2}} \cdot\left(\left\|\sum_{i \leq k} \vec{r}_{i} \cdot t_{i}\right\|+d_{1}\right)\right) \\
& \leq \frac{\gamma}{d_{1}+d_{2}} \cdot\left(d_{2}+d_{1}\right) \leq \gamma
\end{aligned}
$$

and so any point reachable under $\sigma^{\prime}$ is within the distance $\gamma$ from the initial point, and hence in the safety set. Consequently, there is a safe CMS subsystem, which is a contradiction.

The above negative observations imply that to solve the schedulability games for BMS one needs to take a different approach. In the rest of this section, we define the notion of $\mathcal{H}$-closed polytope and show that if such a polytope exists, then for any convex set $S$ we can construct a winning dynamic strategy which takes its decisions only based on the last state. We also extend the notion of safety of a CMS to BMS. We say that a BMS $\mathcal{H}$ is safe if all instances of its extreme-rate MMS $\operatorname{Ext}(\mathcal{H})$ are safe, i.e. all $H \in \llbracket \operatorname{Ext}(\mathcal{H}) \rrbracket$ satisfy (1). Finally, we connect (in Lemmas 3.4 and 3.5 the existence of $\mathcal{H}$-closed polytope with the safety of the BMS.

Dynamic Scheduling Algorithm. For a BMS $\mathcal{H}$ we call a convex polytope $P \mathcal{H}$-closed, if for every vertex of $P$ there exists a mode $m$ such that all the rate vectors of $m$ keep the system in $P$, i.e. for all $\bar{c} \in \operatorname{vert}(P)$ there exists $m \in M$ and $\tau \in \mathbb{R}_{>0}$ such that for all $\vec{r} \in \mathcal{R}(m)$ and all $t \in[0, \tau]$ we have $\bar{c}+\vec{r} \cdot t \in P$. An example of a $\mathcal{H}$-closed polytope is given in the Example 1.4.

Assume that for any $\gamma>0$ and $\bar{x}_{0}$ we are able to compute a $\mathcal{H}$-closed polytope which is fully contained in $B_{\gamma}\left(\bar{x}_{0}\right)$ and contains $\bar{x}_{0}$. If this is the case, we can use Algorithm 1 to compute a dynamic scheduling strategy. The idea of the algorithm is to use the $\mathcal{H}$-closed polytope which contains the initial state and is fully contained within $S$, and then construct the strategy based on the modes safe at the vertices of the polytope. The correctness of the algorithm is established by the following proposition.

Proposition 3.3. If there exists an $\mathcal{H}$-closed polytope and it can be effectively computed then Algorithm 1 implements a winning dynamic strategy for the scheduler. 


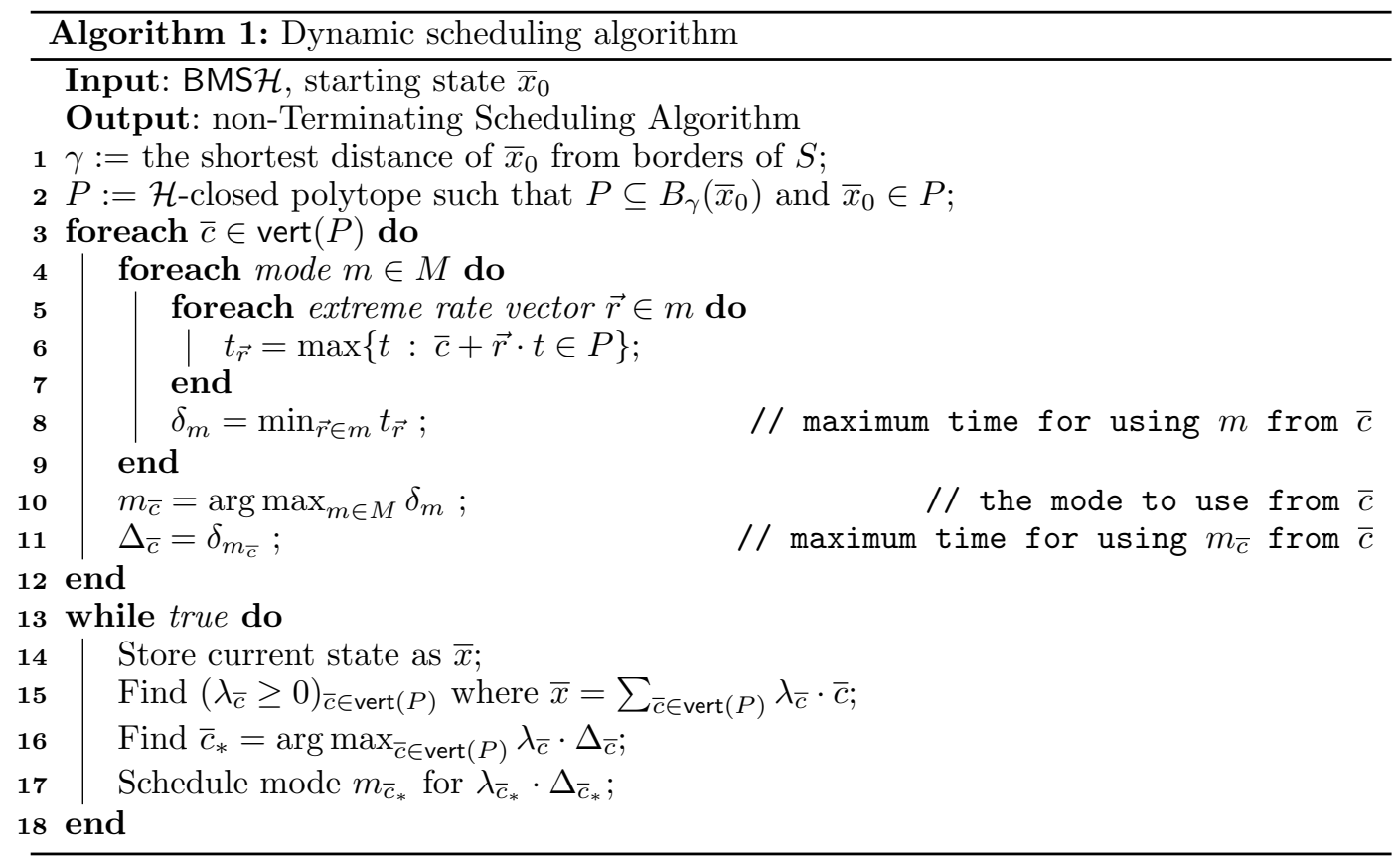

Proof. Assume that there exists an $\mathcal{H}$-closed polytope and we have an algorithm to compute it. Observe that the strategy implemented by the algorithm is non-Zeno, because $\lambda_{\bar{c}_{*}} \cdot \Delta_{\bar{c}_{*}}$ on line 17 is bounded from below by $\frac{1}{|\operatorname{vert}(P)|} \cdot \min _{\bar{c} \in \operatorname{vert}(P)} \Delta_{\bar{c}}$ for any point of $P$, and $\Delta_{\bar{c}}$ are positive by their construction and the definition of the $\mathcal{H}$-closed polytope. Next, we need to show that under the computed strategy we never leave the convex polytope $P$. For a state $\bar{x}$ which is of the form $\sum_{\bar{c} \in \operatorname{vert}(P)} \lambda_{\bar{c}} \cdot \bar{c}$, the successor state will be $\bar{x}^{\prime}=$ $\left(\sum_{\bar{c} \in \operatorname{vert}(P)} \lambda_{\bar{c}} \cdot \bar{c}\right)+\lambda_{\bar{c}_{*}} \cdot \Delta_{\bar{c}_{*}} \cdot \vec{r}$ where $\vec{r}$ is the rate picked by the environment. We can rewrite $\bar{x}^{\prime}$ as $\left(\sum_{\bar{c} \in \operatorname{vert}(P) \backslash\left\{\bar{c}_{*}\right\}} \lambda_{\bar{c}} \cdot \bar{c}\right)+\lambda_{\bar{c}_{*}} \cdot\left(\bar{c}_{*}+\vec{r} \cdot \Delta_{\bar{c}_{*}}\right)$. Since $\bar{c}_{*}+\vec{r} \cdot \Delta_{\bar{c}_{*}} \in P$, we get that $\bar{x}^{\prime}$ is a convex combination of points in $P$ and hence lies in $P$.

Constructing $\mathcal{H}$-Closed Polytope. We will next show how to implement line 2 of Algorithm 1. We give necessary and sufficient conditions for existence of $\mathcal{H}$-closed polytopes in the following two lemmas. The first lemma shows that an $\mathcal{H}$-closed polytope exists if and only if for any hyperplane (given by its normal vector $\vec{v}$ ) there exists a mode $m$ such that all its rates stay at one side of the hyperplane.

Lemma 3.4. For a BMS $\mathcal{H}$, a state $\bar{x}_{0}$ and $\gamma>0$, there is a $\mathcal{H}$-closed polytope $P \subseteq$ $B_{\gamma}\left(\bar{x}_{0}\right)$ with $\bar{x}_{0} \in P$ if and only if for every $\vec{v}$ there is a mode $m$ such that $\vec{v} \cdot \vec{r} \geq 0$ for all $\vec{r} \in m$.

Proof. Let us fix a BMS $\mathcal{H}=(M, n, \mathcal{R})$. The proof is in two parts. For $\Rightarrow$, assume that the system is schedulable but there exists a vector $\vec{v}$ such that for all modes $m \in M$ there is a rate $\vec{r}_{m} \in m$ where $\vec{v} \cdot \vec{r}_{m}<0$. It implies that if the adversary fixes the rates $\vec{r}_{m}$ whenever the scheduler chooses $m$, then the system moves in the direction of vector $-\vec{v}$ (i.e. for all $d$ a state $\bar{x}$ will be reached such that $\vec{v} \cdot \bar{x}<d$ ), and hence for any bounded safety set and non-Zeno strategy system will leave the safety set. This contradicts with existence of $\mathcal{H}$-closed polytope, which itself implies the existence of a winning scheduler strategy. 


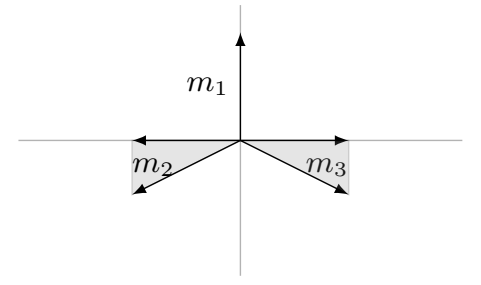

(a)

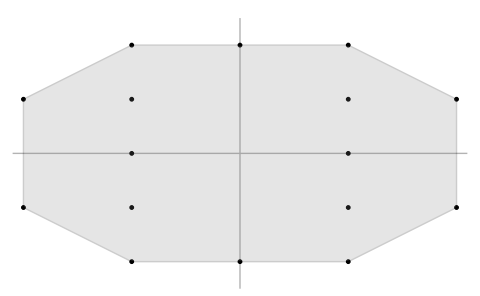

(c)

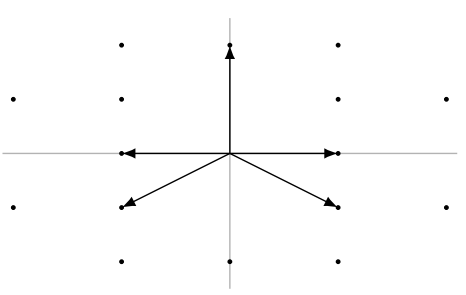

(b)

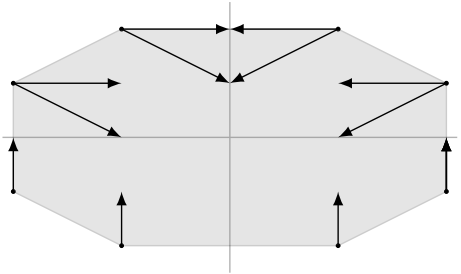

(d)

Fig. 4. Constructing closed convex polytope

To prove the other direction, let $R=\left\{\vec{r}_{1}, \ldots, \vec{r}_{N}\right\}$ be the set of rates occurring in modes of the extreme-rate MMS of $\mathcal{H}$, i.e. $R=\left\{\mathcal{R}^{\prime}(m) \mid\left(M, n, \mathcal{R}^{\prime}\right) \in \llbracket \operatorname{Ext}(\mathcal{H}) \rrbracket, m \in M\right\}$. We claim the following to be an $\mathcal{H}$-closed polytope:

$$
P:=\left\{\bar{x}_{0}+D \cdot \sum_{i=1}^{N} \vec{r}_{i} \cdot p_{i} \mid p_{i} \in[0,1]\right\},
$$

where $D=\gamma / \sum_{i=1}^{N}\left\|\vec{r}_{i}\right\|$. Notice that $P$ is a convex polytope since it is a convex hull of points $\bar{x}_{0}+D \cdot \sum_{i=1}^{N} \vec{r}_{i} \cdot p_{i}$ where $p_{i} \in\{0,1\}$. Also, due to our choice of $D, P \subseteq B_{\gamma}\left(\bar{x}_{0}\right)$, and $\bar{x}_{0} \in P$. For the sake of contradiction we assume that for every $\vec{v}$ there is a mode $m$ such that all rates $\vec{r}$ of $m$ satisfy $\vec{v} \cdot \vec{r} \geq 0$, but at least one corner $\bar{c}$ of $P$ does not satisfy the defining condition of $\mathcal{H}$-closed polytope, i.e. for all modes $i$ there is a rate vector $\vec{r}_{i}$ satisfying

$$
\bar{c}+t \cdot \vec{r}_{i} \notin P \text { for all } t>0
$$

Let us fix such corner $\bar{c}$. By the supporting hyperplane theorem $[6]$ there is a vector $\vec{v}$ such that, for some $d$ :

$$
\begin{aligned}
& \vec{v} \cdot \bar{c}=d \\
& \vec{v} \cdot \bar{x}>d, \text { for all } \bar{x} \in P \backslash\{\bar{c}\}
\end{aligned}
$$

i.e. $\vec{v}$ is supporting $P$ on $\bar{c}$. Let us fix some mode $m$ such that for all rates $\vec{r}$ of $m$ we have $\vec{v} \cdot \vec{r} \geq 0$. Such mode exists by the assumption. Let $\vec{r}_{i}$ be a rate of $m$ satisfying (3).

By the definition of $P$ the point $\bar{c}$, a corner of $P$, is of the form $\bar{x}_{0}+D \cdot \sum_{j=1}^{N} \vec{r}_{j} \cdot p_{j}$ for some $p_{j} \in[0,1]$ where $1 \leq j \leq N$ and $\vec{r}_{j} \in R$. We necessarily have $p_{i}=1$, because if $p_{i}=1-\delta$ for some $\delta>0$, then $\bar{c}+D \cdot \varepsilon \cdot \vec{r}_{i} \in P$ for any $\varepsilon \leq \delta$ and that will contradict with (3). Notice that for all $k \in[0,1]$ the points

$$
\bar{y}_{k}=\bar{x}_{0}+D \cdot \sum_{j=1}^{N} p_{j}^{k} \cdot \vec{r}_{j} \quad \text { where } p_{j}^{k}=p_{j} \text { if } j \neq i \text { and } p_{j}^{k}=k \text { otherwise }
$$




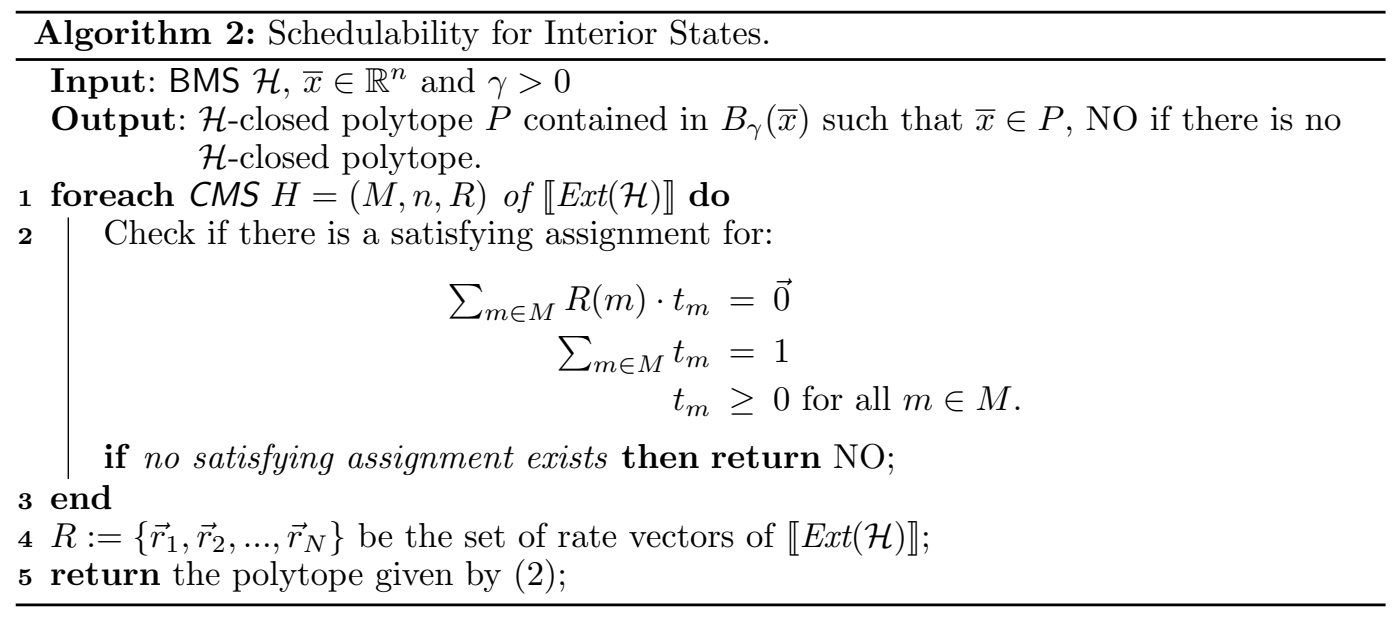

are all in $P$. Further, $\bar{y}_{1}=\bar{c}$ and for each $k \in[0,1]$ we have that $\bar{y}_{k}=\bar{y}_{0}+D \cdot k \cdot \vec{r}_{i}$. In particular, $\bar{c}=\bar{y}_{1}=\bar{y}_{0}+D \cdot \vec{r}_{i}$. It follows that $\bar{c}-D \cdot \vec{r}_{i}=\bar{y}_{0} \in P$. We get

$$
\begin{array}{rr}
\vec{v} \cdot\left(\bar{c}-D \cdot \vec{r}_{i}\right)>d & \left(\text { by }(5), \text { and because } \vec{r}_{i} \neq \overrightarrow{0} \text { by }(3) \text { and so } \bar{c}-D \cdot \vec{r}_{i} \neq \bar{c}\right. \text {.) } \\
\vec{v} \cdot \bar{c}-D \cdot \vec{v} \cdot \vec{r}_{i}>d & (\text { rearranging) } \\
D \cdot \vec{v} \cdot \vec{r}_{i}<0 & \text { (because } \vec{v} \cdot \bar{c}=d \text { by (4)) }
\end{array}
$$

As $D$ is positive, this contradicts that $\vec{v} \cdot \vec{r}_{i} \geq 0$.

Figures 4.(b)-(c) show how to construct $\mathcal{H}$-closed polytope from (2) for the BMS in Figure 4(a), while Figure 4.(d) shows that for every corner of the constructed polytope there is a mode that keeps the system inside the polytope.

The following lemma finally gives an algorithmically checkable characterization of existence of $\mathcal{H}$-closed polytope.

LEMma 3.5. Let $\mathcal{H}=(M, n, \mathcal{R})$ be a BMS. $\mathcal{H}$ is safe if and only if for every $\vec{v}$ there is a mode $m$ such that $\vec{v} \cdot \vec{r} \geq 0$ for all $\vec{r} \in m$.

Proof. In the direction $\Leftarrow$, let us assume that $(M, n, R) \in \llbracket E x t(\mathcal{H}) \rrbracket$ is not safe, and let $Q=\{R(m) \mid m \in M\}$. Then $\overrightarrow{0}$ is not a convex combination of points in $Q$, and so by the supporting hyperplane theorem applied to $\overrightarrow{0}$ and the convex hull of $Q$ there is $\vec{u}$ and $d>0$ such that $\vec{u} \cdot R(m) \geq d$ for all $m \in M$. Since $R(m) \in \mathcal{R}(m)$, this direction of the proof is finished by putting $\vec{v}=-\vec{u}$.

In the other direction, let $\vec{v}$ be such that for all $m \in M$ there is $\vec{r} \in \mathcal{R}(m)$ satisfying $\vec{v} \cdot \vec{r}<0$. Then by convexity of $\mathcal{R}(m)$ there is $\vec{r}_{m} \in \operatorname{vert}(\mathcal{R}(m))$ with the same properties, and we can create a CMS $(M, n, R) \in \llbracket E x t(\mathcal{H}) \rrbracket$ by putting $R(m)=\vec{r}_{m}$. This CMS is not safe, because for any strategy, for a sufficiently large time bound a point $\bar{x}$ will be reached such that $(-\vec{v}) \cdot \bar{x}$ is arbitrarily large, and hence any convex polytope will be left eventually.

Combining Proposition 3.3 with Lemmas 3.4 and 3.5 we get the following main result.

THEOREM 3.6. For every BMS $\mathcal{H}$ and the starting state in the interior of a convex and bounded safety set we have that the scheduler has a winning strategy if and only if $\mathcal{H}$ is safe.

Theorem 3.6 allows us to devise Algorithm 2 and at the same time give its correctness. The reader may have noticed that Theorem 3.6 bears a striking resemblance to Theorem 3.1 for CMS, since the former boils down to checking safety of exponentially many CMS instances. 


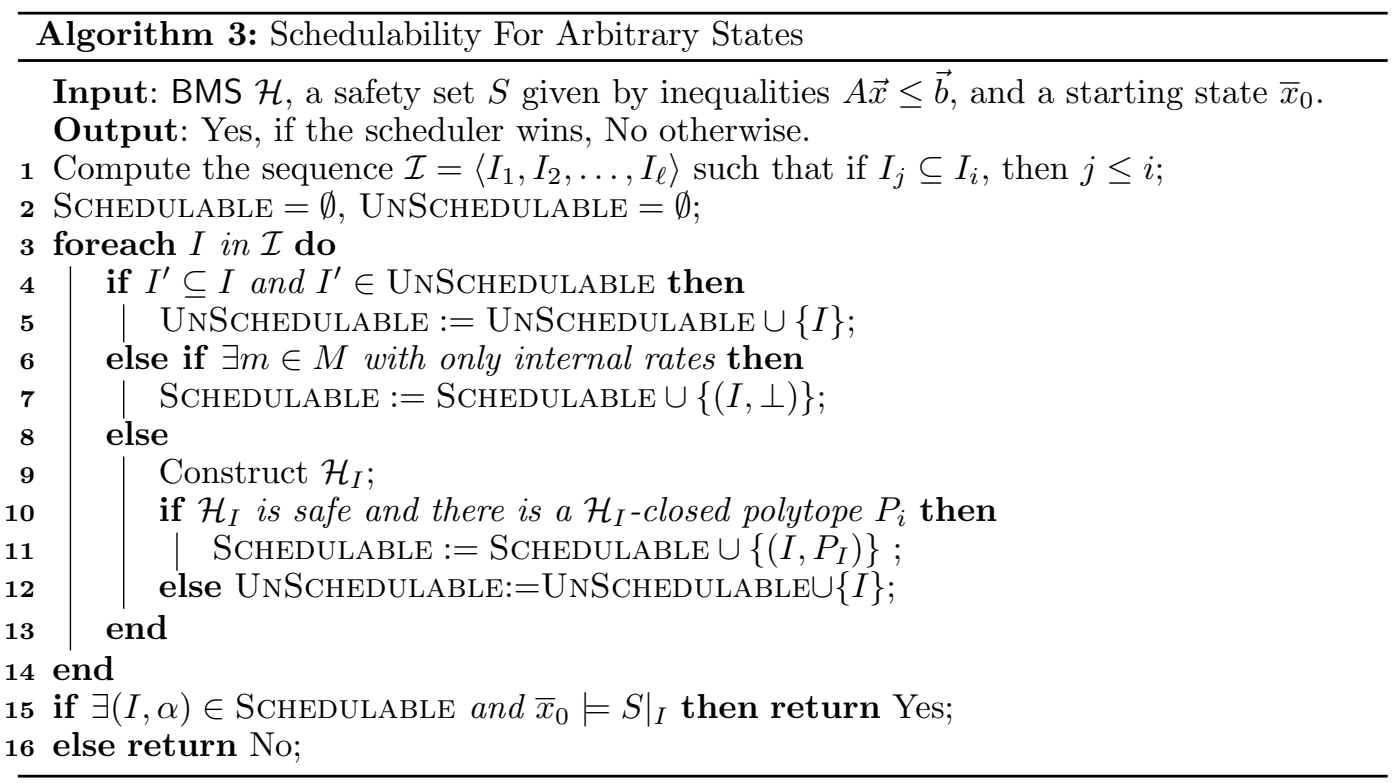

Note, however, that the proof here is much more delicate. While in the case of CMS satisfaction of (1) gives immediately a periodic winning strategy, for BMS this is not the case: even when every instance in $\llbracket E x t(\mathcal{H}) \rrbracket$ is safe, we cannot immediately see which modes should be used by the winning strategy; this requires the introduction of $\mathcal{H}$-closed polytopes.

\subsection{General Case}

In this section we present Algorithm 3 that analyses schedulability of arbitrary starting states in $S$. Notice that a starting state on the boundary of the safety polytope may lie on various faces (planes, edges etc.) of different dimensions. The scheduler may have a winning strategy using modes that let the system stay on some lower dimension face, or there may exists a winning strategy where the scheduler first reaches a face of higher dimension where it may have a winning strategy. Before we describe steps of our algorithm, we need to formalize a notion of (open) faces of a convex polytope, a concept critical in Algorithm 3

Let $A x \leq b$ be the linear constraints specifying a convex polytope $S$. We specify a face of $S$ by a set $I \subseteq\{1, \ldots, \operatorname{rows}(A)\}$. We write $\bar{x}=\left.S\right|_{I}$, and we say that $\bar{x}$ satisfies $\left.S\right|_{I}$, if and only if $A_{1, j} x(1)+\cdots A_{n, j} x(n)=b_{j}$ for all $j \in I$, and $A_{1, j} x(1)+\cdots A_{n, j} x(n)<b_{j}$ for all $j \notin I$, i.e. exactly the inequalities indexed by numbers from $I$ are satisfied tightly. Note that every point of $S$ satisfies $\left.S\right|_{I}$ for exactly one $I$. Although there are potentially uncountably many states in every face of $S$, the following Lemma implies that it is sufficient to analyze only one state in every face.

Lemma 3.7. Let $\mathcal{H}=(M, n, \mathcal{R})$ be a BMS, $S$ be a convex polytope, $I$ and $I^{\prime}$ two faces of $S$ such that $I^{\prime} \subseteq I$, and let $\bar{x}$ and $\bar{y}$ be two points satisfying $\left.S\right|_{I}$ and $\left.S\right|_{I^{\prime}}$, respectively. Then $\bar{y}$ is schedulable whenever $\bar{x}$ is schedulable.

Proof. Assume $\bar{x}$ is safe with a strategy $\sigma$, and let $\gamma$ be a non-zero number such that when playing according to $\sigma$ from $\bar{x}$, no point farther than $\gamma$ from $\bar{x}$ is reached. Let $\beta$ be the distance of $\bar{y}$ to any point $\bar{y}^{\prime}$ satisfying $\left.\bar{y}^{\prime} \models S\right|_{I^{\prime \prime}}$ for $I^{\prime \prime} \nsubseteq I^{\prime}$; we necessarily have $\beta>0$. We put $d=\gamma / \beta$, and define a strategy $\sigma^{\prime}$ for the controller, witnessing that $\bar{y}$ is schedulable. Intuitively, $\sigma^{\prime}$ will assume that the starting point is $\bar{y}$ rather than $\bar{x}$, and in addition the time steps will be scaled down by $d$ to make sure the safety set is never left. 
Formally, let $\varrho=\left\langle\bar{y}_{0},\left(m_{1}, t_{1}\right), \vec{r}_{1}, \bar{y}_{1}, \ldots \bar{y}_{k}\right\rangle$ where $\bar{y}_{i}=\bar{y}+\bar{y}_{i}^{\prime}$ for some $\bar{y}_{i}^{\prime}$ be a history, and let $(m, t \cdot d)$ be a decision of $\sigma$ on

$$
\left(\bar{x}+\bar{y}_{0}^{\prime} \cdot d,\left(m_{1}, t_{1} \cdot d\right), \vec{r}_{1}, \bar{x}+\bar{y}_{1}^{\prime} \cdot d,\left(m_{2}, t_{2} \cdot d\right), \vec{r}_{2}, \bar{x}+\bar{y}_{2}^{\prime} \cdot d \ldots \bar{x}+\bar{y}_{k} \cdot d\right) .
$$

The strategy $\sigma^{\prime}$ chooses $(m, t)$ in $\varrho$. We argue that when starting in $\bar{y}$ the strategy $\sigma^{\prime}$ will never allow a point not outside of $S$ to be reached. Because $I^{\prime} \subseteq I$ and due to the properties of $\sigma$, all points $\bar{z}$ reachable will satisfy $A_{1, j} x(1)+\cdots A_{n, j} x(n) \leq b_{j}$ for all $j \in I$. Then, reaching any point outside of $S$ would require "crossing" $S$ in a point $\bar{z}$ such that $A_{1, j} x(1)+\cdots A_{n, j} x(n)=b_{j}$ for some $j \notin I$. However, this is not possible due to the properties of $\beta$ and $I^{\prime \prime}$ (and subsequently choice of $d$ ).

Let $\mathcal{I}=\left\langle I_{1}, I_{2}, \ldots\right\rangle$ be the sequence of all faces such that $\left.S\right|_{I_{i}}$ is satisfied by some state, ordered such that if $I_{i} \subseteq I_{j}$, then $i \leq j$. We call a rate $\vec{r}$ external for $I$ if there is $\left.\bar{x} \models S\right|_{I}$ such that $\bar{x}+\vec{r} \cdot \delta \notin S$ for all $\delta>0$. A rate $\vec{r}$ is called internal if for any $\bar{x}$ such that $\bar{x}=\left.S\right|_{I}$ there is $\delta>0$ and $I^{\prime} \subseteq I$ with $\bar{x}+\vec{r} \cdot \varepsilon=\left.S\right|_{I^{\prime}}$ for all $0<\varepsilon \leq \delta$. For a BMS $\mathcal{H}$ and face $I$ we define a BMS $\mathcal{H}_{I}=\left(M_{I}, n, \mathcal{R}^{\prime}\right)$ where $M_{I}$ contains all modes of $M$ which do not contain rates external for $I$, and $\mathcal{R}^{\prime}(m)$ is the set of non-internal rates of $\mathcal{R}(m)$.

THEOREM 3.8. For every BMS $\mathcal{H}$, a convex polytope safety set $S$, and a starting state $\bar{x}_{0} \in S$, Algorithm 3 decides schedulability problem for $\mathcal{H}$. Moreover, one can construct a dynamic winning strategy using the set SCHEDULABLE.

Proof. (Sketch.) Let $\left\langle I_{1}, I_{2}, \ldots\right\rangle$ be all sets such that $\left.S\right|_{I_{i}}$ is satisfied by some state, ordered such that if $I_{i} \subseteq I_{j}$, then $i \leq j$. Algorithm 3 analyzes the sets $I_{i}$, determining whether the points satisfying $\left.S\right|_{I_{i}}$ are schedulable (in which case we call $I_{i}$ schedulable), or not. Let us assume that $I$ is the first element of the sequence $\left\langle I_{1}, I_{2} \ldots\right\rangle$ which has not been analyzed yet. If there is $I^{\prime}$ such that $I^{\prime} \subseteq I$ and $I^{\prime}$ is already marked as not schedulable, then by Lemma 3.7 one can immediately mark $I$ as non-schedulable. If all modes contain external rates, then no point $\bar{x}$ such that $\left.S\right|_{I}$ is schedulable. Notice that if there exists an internal rate to face $I_{j}$ then it must necessarily be the case that $I_{j}$ is schedulable. If there is a mode $m$ which only has internal rates, there is a winning strategy $\sigma$ for the scheduler which starts by picking $m$ and a sufficiently small time interval $t$. This will make sure that after one step a point is reached which is already known to be schedulable and the scheduler has a winning strategy.

If none of the previous cases match, the algorithm creates a BMS $\mathcal{H}_{I}$ and applies Theorem 3.6 to the system $\mathcal{H}_{I}$. If there is a $\mathcal{H}_{I}$-closed polyhedron $P$, we know that $I$ is schedulable and give a winning scheduler's strategy $\sigma_{\bar{x}}$ for any point $\left.\bar{x} \models S\right|_{I}$ as follows. Let $d>0$ be a number such that for any $\bar{y}=I_{j}$ where $j>i$ we have $\|\bar{x}, \bar{y}\|>d$, i.e. $d$ is chosen so that all points of $S$ contained in $B_{d}(\bar{y})$ satisfy $\left.S\right|_{I^{\prime}}$ for $I^{\prime} \subseteq I$ (this follows from the properties of the sequence $I_{1}, I_{2}, \ldots$ and because $S$ is a convex polytope). The strategy $\sigma_{\bar{x}}$ works as follows. Let $\varrho$ be a history of the form

$$
\left(\bar{x}_{0},\left(m_{1}, t_{1}\right), \vec{r}_{1}, \bar{x}_{1} \ldots \bar{x}_{k}\right)
$$

and let $I^{\prime}$ be such that $\bar{x}_{k}=\left.S\right|_{I^{\prime}}$, and let $\ell$ be the smallest number such that for all $\ell \leq \ell^{\prime} \leq k$ we have $\left.\bar{x}_{\ell^{\prime}} \models S\right|_{I^{\prime}}$. If $\left(I^{\prime}, \perp\right) \in$ SCHEdulable, then $\sigma(\varrho)$ is defined to choose the witnessing mode $m$ (see line 6 for sufficiently small time delay). Otherwise, if $\left(I^{\prime}, P\right) \in$ Schedulable for some polytope $P$, then $\sigma_{\bar{x}}$ behaves in $\varrho$ same as the strategy $\sigma$ obtained in Algorithm 1 for the $\mathcal{H}_{I^{\prime}}$-closed polytope $P$ behaves on the history $\left(\bar{x}_{\ell},\left(m_{\ell+1}, t_{\ell+1}\right), \vec{r}_{\ell+1}, \bar{x}_{\ell+1} \ldots \bar{x}_{k}\right)$.

If $\mathcal{H}_{I}$ is not schedulable for any set and any point, then it is easy to see that for no point satisfying $\left.S\right|_{I}$ there is a schedulable strategy. Indeed, for any strategy $\sigma$, as long as $\sigma$ picks the modes from $M_{I}$, the environment can play a counter-strategy showing that $\mathcal{H}_{I}$ is not schedulable. When any mode from $m \in M \backslash M_{I}$ is used by $\sigma$, we have that $m$ contains an 
external rate and so the environment can pick it: this will ensure reaching a point outside $S$. Hence, we can mark $I$ as unschedulable.

As a consequence of the results just presented, we obtain the following corollary.

\section{Corollary 3.9. Schedulability games are determined.}

Proof. We have shown that if the scheduler does not have a winning strategy, the environment is able to design a winning strategy that selects one particular extreme-rate vector for each mode.

\section{COMPLEXITY}

In this section we analyze complexity of the schedulability problem for BMS. We begin by showing that in general it is co-NP-complete, however it can be solved in polynomial time if the system has only a fixed number of variables.

\subsection{General Case}

The upper complexity bound for the general case is formalised by the following proposition.

Proposition 4.1. The schedulability problem for BMS and convex polytope safety sets is in co-NP.

Proof Sketch. We show that when the answer to the problem of schedulability of a point $\bar{x}$ is No, there is a polynomially checkable falsifier. Denote $I^{\prime}$ the set such that $\bar{x}=\left.S\right|_{I^{\prime}}$. The falsifier is given by a rate combination $\left(\vec{r}_{m}\right)_{m \in M}$ and a set of modes External $\subseteq M$ which satisfy the following conditions.

— there is a vector $\vec{v}$ such that $\vec{v} \cdot \vec{r}_{m}>0$ for all $m \notin$ External.

- for the set

$$
I=\left\{i \in I^{\prime} \mid \forall m \in M \backslash \text { External, } \delta \in \mathbb{R}: A_{1, i} \vec{r}_{m}(1)+\cdots+A_{n, i} \vec{r}_{m}(n)=0\right\}
$$

we have that every $\vec{r}_{m}$ for $m \in$ External is external. Intuitively, $I$ is the set of all hyperplanes that form the facet $\left.S\right|_{I^{\prime}}$ and at the same time are parallel to every $\vec{r}_{m}$.

Firstly, the falsifier is polynomially checkable. The most demanding part, finding a suitable vector $\vec{v}$, can easily be performed by solving a set of linear constraints of polynomial size. The set $I$ can also be easily computed, since for a fixed $m$ the defining condition is satisfied for all $\delta$ whenever it is satisfied for some (arbitrarily picked) non-zero $\delta$.

Let us now show that the existence of this falsifier implies that the answer to the problem is No. Indeed, as long as a strategy of the scheduler keeps using modes $m \notin$ External, the environment can pick the rates $\vec{r}_{m}$, and a point outside of $S$ will be reached under any nonZeno strategy, due to the properties of $\vec{v}$ and Lemma 3.5. If the strategy of the scheduler picks any mode $m \in$ External, the environment can win immediately by picking the external rate $\vec{r}_{m}$; because $I^{\prime} \subseteq I$, this will ensure that a point outside $S$ is reached.

On the other hand, let us suppose that the answer to the problem is No, and let $I^{\prime}$ be such that $\bar{x}=\left.S\right|_{I^{\prime}}$. Then consider any minimal non-schedulable $I \subseteq I^{\prime}$. We put to External all modes $m$ which contain rates external for $I$, and for every such mode we select an external rate $\vec{r}_{m}$. Further, there is not any mode with only internal rates (otherwise $I$ would not be minimal non-schedulable) and the BMS $\mathcal{H}_{I}$ must be non-schedulable (otherwise $I$ would be schedulable). By Theorem 3.6 there is an unsafe instance $H=\left(M^{\prime}, n, R\right) \in \llbracket \operatorname{Ext}\left(\mathcal{H}_{I}\right) \rrbracket$. Since $M^{\prime}=M \backslash$ External, we can pick the rates $\vec{r}_{m}$ for every $m \in M^{\prime}$ to be the rates of this unsafe instance.

We will now argue that the rates $\left(\vec{r}_{m}\right)_{m \in M}$ and the set External give rise to a valid falsifier. Firstly, we pick $\vec{v}$ to be the vector showing that $\left(M^{\prime}, n, R\right)$ is an unsafe instance. Secondly, 

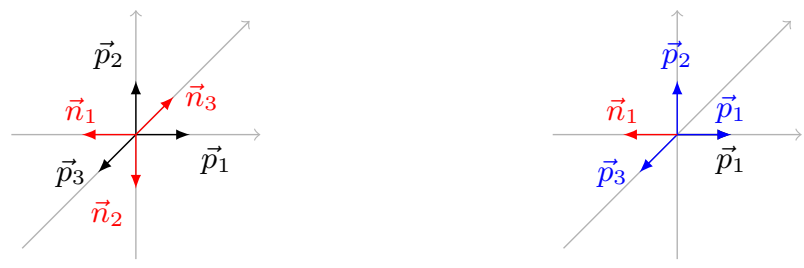

Fig. 5. An example from the proof of Proposition 4.2

let $X:=\left\{i \in I^{\prime} \mid \forall m \in M \backslash\right.$ External, $\left.\delta \in \mathbb{R}: A_{1, i} \vec{r}_{m}(1)+\cdots+A_{n, i} \vec{r}_{m}(n)=0\right\}$, and note that for all $i \in I$, all $m \in M \backslash$ External and all $\delta$ we have $A_{1, i} \vec{r}_{m}(1)+\cdots+A_{n, i} \vec{r}_{m}(n)=0$ by definition of $\mathcal{H}_{I}$, and so $I \subseteq X$. Then, because any rate that is external for $I$ is also external for any superset of $I$, we have that $\vec{r}_{m}$ is external for all $m \in$ External.

Proposition 4.2 (CO-NP HARDNESs). The schedulability problem for MMS is co-NP hard.

Proof. The proof for co-NP hardness uses a reduction from the classical NP-complete problem 3-SAT. For a SAT instance $\phi$ we construct a MMS $\mathcal{H}_{\phi}$ such that $\phi$ is satisfiable if and only if $\mathcal{H}_{\phi}$ is not schedulable for any starting state and bounded convex safety set. Consider a SAT instance $\phi$ with $k$ clauses and $n$ variables denoted as $x_{1}, \ldots, x_{n}$. The corresponding MMS $\mathcal{H}_{\phi}=(M, n, \mathcal{R})$ is such that its set of modes $M=\left\{m_{1}, \ldots, m_{k}\right\}$ corresponds to the clauses in $\phi$, and variables are such that variable $i$ corresponds to variable $x_{i}$ of $\phi$. For each variable $x_{i}$ we define vectors $\vec{p}_{i}$ and $\vec{n}_{i}$ such that $\vec{p}_{i}(i)=1, \vec{n}_{i}(i)=-1$, and $\vec{p}_{i}(j)=\vec{n}_{i}(j)=0$ if $i \neq j$. The rate-vector function $\mathcal{R}$ is defined such that for each mode $m_{j}$ and for each SAT variable $x_{i}$ we have that $\vec{p}_{i} \in \mathcal{R}\left(m_{j}\right)$ if $x_{i}$ occurs positively in clause $j$, and $\vec{n}_{i} \in \mathcal{R}\left(m_{j}\right)$ if the variable $x_{i}$ occurs negatively in clause $j$. The crucial property here is that there is no vector that can have a positive dot product with both $\vec{p}_{i}$ and $\vec{n}_{i}$, which allows us to map unsafe rate combinations to satisfying valuations and vice versa. Figure 5 shows an example of the reduction for two different formulas. On the left, we have a satisfiable formula $\left(x_{1} \vee x_{2} \vee x_{3}\right) \wedge\left(\neg x_{1} \vee \neg x_{2} \vee \neg x_{3}\right)$ which gives rise to a MMS with two modes: $\left\{\vec{p}_{1}, \vec{p}_{2}, \vec{p}_{3}\right\} \in m_{1}$ and $\left\{\vec{n}_{1}, \vec{n}_{2}, \vec{n}_{3}\right\} \in m_{2}$. The system has unsafe combination $\vec{p}_{1}, \vec{n}_{2}$. In Figure 5 (right) an unsatisfiable formula $\left(x_{1} \vee x_{1} \vee x_{1}\right) \wedge\left(\neg x_{1} \vee \neg x_{1} \vee \neg x_{1}\right) \wedge\left(x_{1} \vee x_{2} \vee x_{3}\right)$ is reduced to a MMS with three modes: $\left\{\vec{p}_{1}\right\} \in m_{1},\left\{\vec{n}_{1}\right\} \in m_{2}$, and $\left\{\vec{p}_{1}, \vec{p}_{2}, \vec{p}_{3}\right\} \in m_{3}$. All combinations are safe.

We now proceed with showing that the above reduction is correct, that is, we show that there is a satisfying assignment for $\varphi$ if and only if there exists an unsafe instance of $\mathcal{H}_{\phi}$.

First, let us suppose that there is an unsafe combination $\left\{\vec{r}_{i}\left|\vec{r}_{i} \in m_{i}, 1 \leq i \leq\right| M \mid\right\}$. Then for every rate $\vec{r}_{i}$ which contains 1 at $i$-th position assign true to the variable $x_{i}$, and for every rate $\vec{r}_{i}$ which contains -1 at $i$-th position assign false to the variable $x_{i}$. Note that no variable is assigned both true and false since if two vectors $\vec{r}^{\prime}$ and $\vec{r}^{\prime \prime}$ are chosen which go to the opposite direction, then every $\vec{v}$ which satisfies $\vec{v} \cdot r^{\prime}>0$ also satisfies $\vec{v} \cdot r^{\prime \prime}<0$, and vice versa, which means that the combination is not unsafe. Further, observe that the assignment is satisfying, because for every clause $c_{j}$ we have that if $\vec{r}_{j}$ contains 1 at $i$-th position, then $c_{j}$ contains the literal $x_{i}$ which is satisfied, and if $\vec{r}_{j}$ contains -1 at $i$-th position, then $c_{j}$ contains the literal $\neg x_{i}$ which is satisfied. Hence there is at least one true literal in each clause and thus the formula $\phi$ is satisfiable.

To prove the other direction, assume that there is a satisfying assignment to $\phi$, then choose one true literal from each clause and consider the corresponding rate vector for each mode. Note that there would be no two vectors along one axis with different directions since $\neg x_{i}$ and $x_{i}$ can not be true at the same time. Therefore we have $k$ vectors along $1 \leq d \leq n$ axises where each two vectors are either same or perpendicular. This set of rate vectors will be unsafe since there exists a $\vec{v}$ with strictly positive dot product with all of them: We build 
vector $\vec{v}$ such that each $i$-th entry of vector $\vec{v}$ is 1 (resp. -1 ), if there are some vectors whose $i$-th entry is 1 (resp. -1 ), and zero otherwise. The product of $v$ with any vector from the combination is equal to 1 , and hence greater than zero.

The following corollary is immediate.

COROLlary 4.3 (CO-NP HARDNESS RESUlt FOR BMS). The schedulability problem for BMS is co-NP hard.

ProOF. To prove this corollary we show that if there is an unsafe instance of BMS $\mathcal{H}$ then there is an unsafe instance of corresponding extreme-rate MMS $\operatorname{Ext}(\mathcal{H})$. With this observation, the corollary then follows from Proposition 4.2. Assume $m$ is a mode in the bounded-rate multi-mode system $\mathcal{H}$ with extreme rate vectors $\left\{\vec{r}_{1}^{*}, \ldots, \vec{r}_{k}^{*}\right\}$. First we show that if there is a rate vector $\vec{r} \in m$ and a rate vector $\vec{v}$ such that their dot product is positive, i.e. $\vec{v} \cdot \vec{r}>0$, then there exists at least one extreme rate vector $\vec{r}_{i}^{*}$ with $\vec{v} \cdot \vec{r}_{i}^{*}>0$. Because $\vec{r}=\sum_{i=1}^{k} \lambda_{i} \cdot \vec{r}_{i}^{*}$ for non-negative coefficients $\lambda_{i}$, we have $\vec{v} \cdot \vec{r}=\sum_{i=1}^{k} \lambda_{i} \cdot\left(\vec{v} \cdot \vec{r}_{i}^{*}\right)$ and so for $\vec{v} \cdot \vec{r}$ to be positive, there must be at least one positive summand.

Thus if there is an unsafe instance of BMS, for each mode we can choose a extreme rate such that the corresponding extreme-rate instance is unsafe.

\subsection{BMS with a fixed number of variables}

Now we show that when the number of variables is fixed to $n$, the schedulability problem can be solved in polynomial time. We do this by showing that it is sufficient to consider only polynomially many falsifiers, and so we don't need to rely on an oracle and we can check all candidates for falsifiers in polynomial time.

Given a sequence $\vec{r}_{1}, \ldots, \vec{r}_{d}$ of linearly independent vectors, we use $\operatorname{ort}\left(\vec{r}_{1}, \ldots, \vec{r}_{d}\right)$ for the vector $\vec{u}$ such that

$-\|\vec{u}\|=1$

- $\vec{u}$ is a linear combination of the vectors $\vec{r}_{1}, \ldots, \vec{r}_{d}$

$-\vec{u} \cdot \vec{r}_{1}>0$

$-\vec{u} \cdot \vec{r}_{j}=0$ for all $j>1$

LEMMA 4.4. For any sequence $\vec{r}_{1}, \ldots, \vec{r}_{d}$ of linearly independent vectors, the vector $\operatorname{ort}\left(\vec{r}_{1}, \ldots, \vec{r}_{d}\right)$ exists and is unique.

Proof. Let $V_{1}=\operatorname{span}\left(\vec{r}_{1}, \ldots, \vec{r}_{d}\right)$ and $V_{2}=\operatorname{span}\left(\vec{r}_{2}, \ldots, \vec{r}_{d}\right)$. Because $\operatorname{dim}\left(\vec{r}_{1}, \ldots, \vec{r}_{d}\right)-$ $\operatorname{dim}\left(\vec{r}_{2}, \ldots, \vec{r}_{d}\right)=1$, the orthogonal complement $V_{2}^{\perp}$ of $V_{2}$ in $V_{1}$ has dimension 1 .

There are exactly two vectors, $\vec{v}$ and $\vec{v}^{\prime}$, in $V_{2}^{\perp}$ whose norm is 1 , and they moreover satisfy $\vec{v}=-\vec{v}^{\prime}$. We necessarily have $\vec{v} \cdot \vec{r}_{1} \neq 0$, since otherwise $\vec{v}, \vec{r}_{1}, \ldots, \vec{r}_{d}$ are linearly independent and $\operatorname{span}\left(\vec{v}, \vec{r}_{1}, \ldots, \vec{r}_{d}\right)=V_{1}$, contradicting that $\operatorname{dim}\left(V_{1}\right)=d$. Now if $\vec{v} \cdot \vec{r}_{1}>0, \vec{v}$ is the required vector ort $\left(\vec{r}_{1}, \ldots, \vec{r}_{d}\right)$, otherwise $-\vec{v}$ is the required vector.

The main insight into the solution is given by the following proposition.

Proposition 4.5. Let us have a finite set of vectors $R$ with $\operatorname{dim}(R)=d$. The following two statements are equivalent:

(1) There is $\vec{v}$ such that $\vec{v} \cdot \vec{r}>0$ for all $\vec{r} \in R$.

(2) There are linearly independent vectors $\vec{r}_{1}, \ldots, \vec{r}_{d} \in R$ such that for every $1 \leq i \leq d$ and every

$$
\vec{r} \in R \cap\left(\operatorname{span}\left(\vec{r}_{i}, \ldots, \vec{r}_{d}\right) \backslash \operatorname{span}\left(\vec{r}_{i+1}, \ldots, \vec{r}_{d}\right)\right)
$$

we have $\operatorname{ort}\left(\vec{r}_{i}, \ldots, \vec{r}_{d}\right) \cdot \vec{r}>0$.

Before proving the proposition, we show how it can be utilised to finish the proof. Let us have a MMS $\mathcal{H}=(M, n, \mathcal{R})$ and a point $\bar{x}$ in $S$. We have shown that $\mathcal{H}$ is not schedulable 
in $\bar{x}$ iff there is a counterexample as defined in the proof of Proposition 4.1. In general, finding the counter-example would require trying all possible rate combinations, yielding an algorithm exponential in $|M|$. However we can utilise Proposition 4.5 as follows.

Suppose that there is a counter-example given by $\left(\vec{r}_{m}\right)_{m \in M}$ and a set External, and let $R=\left\{\vec{r}_{m} \mid m \in M \backslash\right.$ External $\}$ and $d=\operatorname{dim}(R)$. Then there is a sequence $\vec{r}_{1} \ldots, \vec{r}_{d}$ of rates satisfying the condition of item 2 . of Proposition 4.5 for $R$. From the sequence we can reconstruct a (possibly different but still valid) falsifier $\left(\vec{r}_{m}^{\prime}\right)_{m \in M}$ and a set External ${ }^{\prime}$ as follows. Let

$$
I=\left\{i \in I^{\prime} \mid \forall 1 \leq j \leq d, \forall \delta \in \mathbb{R}: A_{1, i} \vec{r}_{j}(1)+\cdots+A_{n, i} \vec{r}_{j}(n)=0\right\}
$$

where $I^{\prime}$ is the set such that $\bar{x}=\left.S\right|_{I^{\prime}}$. Note that since

$$
\operatorname{span}\left(\vec{r}_{1} \ldots, \vec{r}_{d}\right)=\operatorname{span}\left(\left(\vec{r}_{m}\right)_{m \in M \backslash \text { External }}\right)
$$

are equal, we also have

$$
I=\left\{i \in I^{\prime} \mid \forall m \in M \backslash \text { External, } \forall \delta \in \mathbb{R}: A_{1, i} \vec{r}_{j}(1)+\cdots+A_{n, i} \vec{r}_{j}(n)=0\right\}
$$

Let $m_{i}$ be a mode to which $\vec{r}_{i}$ belongs, chosen so that $m_{i} \neq m_{j}$ for $i \neq j$. Firstly, we set $\vec{r}_{m_{i}}^{\prime}=\vec{r}_{i}$ for all $1 \leq i \leq d$. Then, we build the set External' and all the other rates $\vec{r}_{m}^{\prime}$ as follows. For every mode $m \in M \backslash\left\{m_{1}, \ldots, m_{d}\right\}$, we check if there is a rate $\vec{r} \in m$ such that for $1 \leq i \leq d$ with $\vec{r} \in \operatorname{span}\left(\vec{r}_{i}, \ldots, \vec{r}_{d}\right) \backslash \operatorname{span}\left(\vec{r}_{i+1}, \ldots, \vec{r}_{d}\right)$ we have ort $\left(\vec{r}_{i}, \ldots, \vec{r}_{d}\right) \cdot \vec{r}>0$. If this is the case, we set $\vec{r}_{m}=\vec{r}$. Otherwise, by the existence of a falsifier and by our choice of $\vec{r}_{1}, \ldots, \vec{r}_{d}$ there must be $\vec{r}^{\prime} \in m$ that is external for $I$, and we set $\vec{r}_{m}=\vec{r}^{\prime}$ and add $m$ to External.

Hence, having a fixed sequence $\vec{r}_{1}, \ldots, \vec{r}_{d}$, we can check in polynomial time whether it gives rise to a valid falsifier. Since there is at most $n \cdot \mathcal{H}^{n}$ candidate sequences, for the fixed $n$ we obtain a polynomial-time algorithm.

Let us now prove Proposition 4.5

Proof of Proposition 4.5. For a set $U$ of vectors, we use

$$
\operatorname{cone}(U)=\left\{\sum_{\vec{r} \in U} \alpha_{\vec{r}} \cdot \vec{r} \mid \forall \vec{r}: \alpha_{\vec{r}} \geq 0\right\}
$$

for the cone spanned by the vectors in $U$. Intuitively, if the condition 1 . is satisfied, then the point $\overline{0}$ is a vertex of cone $(R)$ and we can get the sequence $\vec{r}_{1}, \ldots, \vec{r}_{d}$ from a facet of cone $(R)$. Conversely, for a sequence $\vec{r}_{1}, \ldots, \vec{r}_{d}$ we can consider a supporting hyperplane of the facet determined by the sequence, and slightly change it to get a vector $\vec{v}$ for condition 1 .

Let us now formally prove the direction $1 . \Rightarrow 2$. We rely on a procedure that given a set $U \subseteq R$ containing a finite number of vectors with $\operatorname{dim}(U)=d^{\prime}$ selects a subset $U^{\prime} \subseteq U$ and a vector $r$ such that:

(1) $\operatorname{dim}\left(U^{\prime}\right)=d^{\prime}-1$, and for all $r^{\prime} \in U \cap \operatorname{span}\left(U^{\prime}\right)$ we have $r^{\prime} \in U^{\prime}$.

(2) $r \in U \backslash U^{\prime}$

(3) For any choice $\vec{q}_{1}, \ldots, \vec{q}_{d^{\prime}-1}$ of linearly independent vectors of $U^{\prime}$ and any $\vec{r}^{\prime \prime} \in U \backslash U^{\prime}$ we have $\operatorname{ort}\left(\vec{r}, \vec{q}_{1}, \ldots, \vec{q}_{d^{\prime}-1}\right) \cdot \vec{r}^{\prime \prime}>0$.

We compute sets $U_{1}, \ldots, U_{d+1}$ and vectors $\vec{r}_{1}, \ldots, \vec{r}_{d}$ where $U_{1}=R$, and where $U_{i+1}$ and $\vec{r}_{i}$ are obtained by applying the procedure to $U_{i}$ (note that $U_{d+1}=\emptyset$ ). We claim that the sequence $\vec{r}_{1}, \ldots, \vec{r}_{d}$ is the desired sequence. Indeed, $\vec{r}_{1}, \ldots, \vec{r}_{d}$ are linearly independent, and for any $i$ we have $U_{i}=R \cap \operatorname{span}\left(\vec{r}_{i}, \ldots, \vec{r}_{d}\right)$. Then, the rest follows from item 3 . above 
because

$$
\begin{aligned}
\left(R \cap \operatorname{span}\left(\vec{r}_{i}, \ldots, \vec{r}_{d}\right)\right) \backslash\left(R \cap \operatorname{span}\left(\vec{r}_{i+1}, \ldots, \vec{r}_{d}\right)\right) & \\
& =R \cap\left(\operatorname{span}\left(\vec{r}_{i}, \ldots, \vec{r}_{d}\right) \backslash R \cap \operatorname{span}\left(\vec{r}_{i+1}, \ldots, \vec{r}_{d}\right)\right)
\end{aligned}
$$

We now describe the above procedure. Let $U \subseteq R$ be a set with $\operatorname{dim}(U)=d^{\prime}$. We construct a sequence of vectors $\vec{w}_{0}, \ldots, \vec{w}_{d^{\prime}-1}$ from $\operatorname{span}(U)$ together with a sequence of vectors $\vec{q}_{1}, \ldots, \vec{q}_{d^{\prime}}$ from $U$ such that:

a. $\vec{q}_{1}, \ldots, \vec{q}_{d^{\prime}}$ are linearly independent vectors;

b. $\vec{w}_{i} \cdot \overrightarrow{\vec{q}_{j}}=0$ for all $i>j$; and

c. $\vec{w}_{i} \cdot \vec{r} \geq 0$ for all $i$ and $\vec{r} \in R \backslash \operatorname{span}\left(\vec{q}_{1}, \ldots, \vec{q}_{i}\right)$.

Consequently, we set $\vec{r}=\vec{q}_{d^{\prime}}$ and $U^{\prime}=U \cap \operatorname{span}\left(\vec{q}_{1}, \ldots, \vec{q}_{d^{\prime}}\right)$. Then, for any $\vec{r}^{\prime \prime} \in U \backslash U^{\prime}$ we have $\operatorname{ort}\left(\vec{r}, \vec{q}_{1}, \ldots, \vec{q}_{d^{\prime}-1}\right) \cdot \vec{r}^{\prime \prime}>0$ : the product is non-negative by the last item above, and cannot be equal to 0 as $\operatorname{ort}\left(\vec{r}, \vec{q}_{1}, \ldots, \vec{q}_{d^{\prime}-1}\right) \cdot \vec{q}_{i}=0$ for all $i \leq d^{\prime}-1$, and no vector in a $d^{\prime}$-dimensional space can have $d^{\prime}$ linearly independent orthogonal vectors (i.e. vectors with which it has zero dot product).

Let us now proceed with the construction of $\vec{w}_{0}, \ldots, \vec{w}_{d^{\prime}-1}$ and $\vec{q}_{1}, \ldots, \vec{q}_{d^{\prime}}$. We set $\vec{w}_{0}$ to be any vector of $\operatorname{span}(U)$ such that $\vec{w}_{0} \cdot \vec{r}>0$ for all $\vec{r} \in R$. Such a vector satisfies the conditions b. and c. by definition, but we need to show that it exists in the first place. Let us choose a basis $\vec{b}_{1}, \ldots \vec{b}_{d}$ for the vector space $\operatorname{span}(R)$, such that $\vec{b}_{1}, \ldots \vec{b}_{d^{\prime}}$ is a basis of $\operatorname{span}(U)$, and every $\vec{b}_{i}$ for $i>d^{\prime}$ is orthogonal to all $\vec{b}_{j}$ for $j \leq d^{\prime}$. Let $\alpha_{1}, \ldots \alpha_{d}$ be numbers such that $\vec{v}=\sum_{i=1}^{d} \alpha_{i} \cdot \vec{b}_{i}$, we claim that it suffices to take $\vec{w}_{0}=\sum_{i=1}^{d} \alpha_{i} \cdot \vec{b}_{i}$. Indeed, any $\vec{r} \in U$ is of the form $\sum_{i=1}^{d^{\prime}} \beta_{i} \cdot \vec{b}_{i}$ for some $\beta_{i}$, and we have

$$
\begin{aligned}
\vec{w}_{0} \cdot \vec{r} & =\left(\sum_{i=1}^{d} \alpha_{i} \cdot \vec{b}_{i}\right) \cdot\left(\sum_{j=1}^{d^{\prime}} \beta_{j} \cdot \vec{b}_{j}\right) \\
& =\left(\left(\sum_{i=1}^{d^{\prime}} \alpha_{i} \cdot \vec{b}_{i}\right) \cdot\left(\sum_{i=1}^{d^{\prime}} \beta_{i} \cdot \vec{b}_{i}\right)\right)+\sum_{i=d^{\prime}+1}^{d} \sum_{j=1}^{d^{\prime}} \alpha_{i} \cdot \beta_{i} \vec{b}_{i} \cdot \vec{b}_{j} \\
& =\left(\sum_{i=1}^{d^{\prime}} \alpha_{i} \cdot \vec{b}_{i}\right) \cdot\left(\sum_{i=1}^{d^{\prime}} \beta_{i} \cdot \vec{b}_{i}\right) \quad\left(\vec{b}_{i} \cdot \vec{b}_{j}=0 \text { for } i>d^{\prime} \text { and } j \leq d^{\prime}\right) \\
& =\vec{v} \cdot \vec{r}>0
\end{aligned}
$$

Fix $i \geq 0$ and suppose that we have already defined $\vec{w}_{i}$ and $\vec{q}_{j}$ for $j<i$. Pick $\vec{b} \in$ $U \backslash \operatorname{span}\left(\vec{q}_{1}, \ldots, \vec{q}_{i}\right)$, and pick $\vec{v}_{\perp} \in \operatorname{span}(U)$ that is orthogonal to $\vec{b}$ and all vectors $\vec{q}_{1}, \ldots, \vec{q}_{i}$. Note that for any $p \in[0,1]$, we have $\left(p \cdot \vec{w}_{i}+(1-p) \cdot \vec{v}_{\perp}\right) \cdot \vec{q}_{j}=0$ for all $i \geq j$. Let $p$ be the minimal number such that $\left(p \cdot \vec{w}_{i}+(1-p) \cdot \vec{v}_{\perp}\right) \cdot \vec{q}=0$ for some $\vec{q} \in R \backslash \operatorname{span}\left(\vec{q}_{1}, \ldots, \vec{q}_{i}\right)$; such $p$ must exist: at least for $p=1$ we can substitute $\vec{b}$ for $\vec{q}$.

We put $\vec{w}_{i+1}=\left(p \cdot \vec{w}_{i}+(1-p) \cdot \vec{v}_{Q^{\prime}}\right)$, and $\vec{q}_{i+1}=\vec{q}$ where $\vec{q}$ is any vector of $U \backslash$ $\operatorname{span}\left(\vec{q}_{1}, \ldots, \vec{q}_{i}\right)$ with $\left(p \cdot \vec{w}_{i}+(1-p) \cdot \vec{v}_{\perp}\right) \cdot \vec{q}=0$.

For the direction $2 . \Rightarrow 1$, let $\vec{p}_{i}=\operatorname{ort}\left(\vec{r}_{i}, \ldots, \vec{r}_{d}\right)$ for $1 \leq i \leq d$. We put $\vec{v}=\sum_{j=1}^{d} \alpha_{j} \cdot \vec{p}_{j}$ where $\alpha_{i}$ is defined as follows. Suppose we have defined $\alpha_{1}, \ldots, \alpha_{i-1}$ so that for $\vec{v}_{i-1}=$ $\sum_{j=1}^{i-1} \alpha_{j} \cdot \vec{p}_{j}$ we have $\vec{v}_{i-1} \cdot \vec{r}>0$ for all

$$
\vec{r} \in R \cap\left(\operatorname{span}\left(\vec{r}_{1}, \ldots, \vec{r}_{d}\right) \backslash \operatorname{span}\left(\vec{r}_{i+1}, \ldots, \vec{r}_{d}\right)\right)
$$

Since $R$ is finite, there is $\varepsilon>0$ such that the product $\vec{v}_{i-1} \cdot \vec{r}$ for the $\vec{r}$ above is always at least $\varepsilon$. Also note that for all $\vec{r} \in R \cap \operatorname{span}\left(\vec{r}_{i+1}, \ldots, \vec{r}_{d}\right)$ we necessarily have $\vec{v}_{i-1} \cdot \vec{r}=0$. 
We choose $\alpha_{i}$ so that $\left(\alpha_{i} \cdot \vec{p}_{i}\right) \cdot \vec{r}<\varepsilon$ for all $\vec{r} \in R \cap\left(\operatorname{span}\left(\vec{r}_{1}, \ldots, \vec{r}_{d}\right) \backslash \operatorname{span}\left(\vec{r}_{i+1}, \ldots, \vec{r}_{d}\right)\right)$ Then for every such $\vec{r}$ we have

$$
\left(\sum_{j=1}^{i} \alpha_{j} \cdot \vec{p}_{j}\right) \cdot \vec{r} \geq \varepsilon+\left(\alpha_{j} \cdot \vec{p}_{j}\right) \cdot \vec{r}>0
$$

Further, for every $\left.\vec{r} \in R \cap \operatorname{span}\left(\vec{r}_{i+1}, \ldots, \vec{r}_{d}\right) \backslash \operatorname{span}\left(\vec{r}_{i+2}, \ldots, \vec{r}_{d}\right)\right)$ we have

$$
\left(\sum_{j=1}^{i} \alpha_{j} \cdot \vec{p}_{j}\right) \cdot \vec{r}=\left(\alpha_{j} \cdot \vec{p}_{j}\right) \cdot \vec{r}=\alpha_{j} \cdot\left(\vec{p}_{j} \cdot \vec{r}\right)>0
$$

and for every $\vec{r} \in R \cap \operatorname{span}\left(\vec{r}_{i+2}, \ldots, \vec{r}_{d}\right)$ we have

$$
\left(\sum_{j=1}^{i} \alpha_{j} \cdot \vec{p}_{j}\right) \cdot \vec{r}=0
$$

This finishes the inductive step, and proves that $\vec{v}$ satisfies the required condition.

Example 4.6. Consider an unsafe set of rate vectors $R=\left\{\vec{r}_{1}, \vec{r}_{2}, \vec{r}_{3}, \vec{r}_{4}\right\}$ shown in the following figure in the left-hand side.

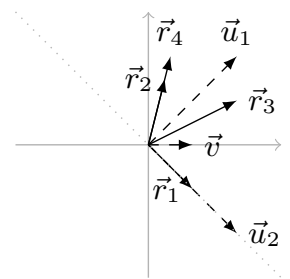

All the rate vectors are on the right side of line $x=0$ and the vector $\vec{v}$ has strictly positive dot product with all of them. We can choose the sequence (of length 2) $\vec{r}_{3}, \vec{r}_{1}$, and see that the vectors $\vec{u}_{1}=\operatorname{ort}\left(\vec{r}_{3}, \vec{r}_{1}\right)$ and $\vec{u}_{2}=\operatorname{ort}\left(\vec{r}_{1}\right)$ satisfy the required conditions: for $\vec{u}_{1}$ which corresponds to the hyperplane marked with a dotted diagonal, we get that no vector lies to the left of the diagonal; for $\vec{u}_{2}$, we only need to consider its dot product with $\vec{r}_{1}$ (as there are no other vectors linearly dependent of $\vec{r}_{1}$ ).

Observe that adding a rate vector $\vec{r}_{5}=-\vec{r}_{1}$ to $R$ makes this set of rate vectors safe, and in particular for the chosen sequence $\vec{r}_{3}, \vec{r}_{1}$ we would have $\operatorname{ort}\left(\vec{r}_{1}\right) \cdot \vec{r}_{5}<0$, thus violating the condition of Proposition 4.5

\section{DISCRETE SCHEDULABILITY}

In this section we discuss the discrete schedulability problem, in which the scheduler can only make decisions at integer multiplies of a specified clock period $\Delta$ and the environment has finitely many choices of rates. Formally, given a MMS $\mathcal{H}$, a closed convex polytope $S$ as safety set, an initial state $x_{0} \in S$, the discrete schedulability problem is to decide if there exists a winning strategy of the scheduler where the time delays are multiples of $\Delta$.

THEOREM 5.1. Discrete schedulability problem is complete for EXPTIME.

Proof. EXPTIME-membership of the problems is shown via discretization of the state space of $\mathcal{H}$. Since the set $S$ is given as a bounded polytope, the size of the discretization can be shown to be at most exponential in the size of $\mathcal{H}$ and $\Delta$, and since the safety games on a finite graph can be solved in P, EXPTIME membership follows.

The hardness is shown by a reduction from the countdown games [11]. A countdown game is a tuple $\mathcal{G}=\left(N, T, n_{1}, B_{1}\right)$ where 
$-N=\left\{n_{1}, n_{2}, \ldots, n_{d}\right\}$ is a finite set of nodes;

$-T \subseteq N \times \mathbb{N}_{>0} \times N$ is a set of transitions; and

$-\left(n_{1}, B_{1}\right) \in N \times \mathbb{N}_{>0}$ is the initial configuration.

From any configuration $(n, B) \in N \times \mathbb{N}_{>0}$, first player 1 chooses a number $k \in \mathbb{N}_{>0}$, such that $k \leq B$ and there exists some $\left(n, k, n^{\prime}\right) \in T$, and then player 2 chooses a transition $\left(n, k, n^{\prime \prime}\right) \in T$ labeled with that number. Note that there can be more than one such transition. The new configuration then transitions to $\left(n^{\prime \prime}, B-k\right)$. Player 1 wins a play of the game when a configuration $(n, 0)$ is reached, and loses (i.e., player 2 wins) when a configuration $(n, B)$ is reached in which player 1 is stuck, i.e., for all transitions $\left(n, k, n^{\prime}\right) \in T$, we have $k>B$.

For a countdown game $\left(N, T, n_{1}, B_{1}\right)$ we define a BMS $\mathcal{H}$, a safety set $S$ and an initial state $\bar{x}$ such that there is a winning scheduler strategy in $\Sigma_{\Delta}$ for $\Delta=1$ iff player 1 has a winning strategy in the countdown game. W.l.o.g we assume that when $\left(n, k, n^{\prime}\right) \in T$, then $n \neq n^{\prime}$, and also we assume that the initial state is $\left(n_{1}, B_{1}\right)$ and there is no node $n$ and $k$ such that $\left(n, k, n_{1}\right) \in T$.

The BMS $\mathcal{H}$ has $d+1$ variables. The intuition is that the value of the first variable corresponds to the value of the counter, while $(i+1)$ th variable is equal to 1 if the game is in node $n_{i}$, and 0 otherwise.

For all $n, k \in N \times \mathbb{N}_{>0}$ such that there is $\left(n, k, n^{\prime}\right) \in T$ for some $n^{\prime}$, we add a mode $(n, k)$ to $\mathcal{H}$. For all $\left(n_{i}, k, n_{j}\right) \in T$, we add the rate $r$ to the mode $\left(n_{i}, k\right)$ such that the first component of $r$ is $-k$, the $(i+1)$ th component is -1 and $(j+1)$ th component is 1 . All other components of $r$ are zero. We further add modes $m_{i}$ for $3 \leq i \leq d+1$ which contain the unique rate with $B_{1}$ in the first component, 1 in the second component, and -1 in $i$-th component. All other components of this rate are zero.

The safety set $S$ is defined so that the only points of $S$ with integer values are exactly $\left(i_{1}, \ldots, i_{d+1}\right)$, where $0 \leq i_{1} \leq B_{1}$, and exactly one of $i_{2}, \ldots, i_{d+1}$ is 1 , while the others are 0 . Such safety set can be defined using equations

$$
\begin{aligned}
x_{1} & \leq B_{1} \\
\sum_{i=2}^{d+1} x_{i} & \leq 1 \\
x_{i} & \geq 0 \quad \text { for } 1 \leq i \leq d+1
\end{aligned}
$$

and so in particular it is convex. Now we claim that the system is schedulable from the point $\left(B_{1}, 1,0,0, \ldots, 0\right)$ iff player 1 has a winning strategy in the countdown game. The intuition is that the winning strategy for player 1 in the countdown game directly gives a strategy for the scheduler in $\mathcal{H}$ such that a point is reached which has zero in the first component, and zeros everywhere else except for some $i$-th component. Then the scheduler uses the mode $m_{i}$, which leads back to the initial state; from there it uses the same strategy. On the other hand, if player 2 has a winning strategy in the countdown game, this strategy can be used to get to a state from which the scheduler has no chance but to leave the safety set (which corresponds to not having any choices in the countdown game).

It is natural to turn the discrete schedulability problem to an optimization problem, by asking to find supremum of all $\Delta$ for which the answer to the discrete schedulability problem is yes. We prove the following, which also improves a result of [2] where only an approximation algorithm was given.

THEOREM 5.2. Given a MMS $\mathcal{H}$, a closed convex polytope $S$ and an initial state $\bar{x}_{0}$, there is an exponential-time algorithm which outputs the supremal $\Delta_{\max }$ for which the answer to the discrete schedulability problem is Yes. For a CMS the algorithm can be constructed to run in polynomial space.

Proof. Let $\Sigma_{\Delta}$ denote the set of strategies for the scheduler which schedule in multiples of $\Delta$. We wish to find a supremum, over all $\Delta$, such that there is a winning strategy in $\Sigma_{\Delta}$. 
Let $R$ be the set of all possible rate vectors of $\mathcal{H}$. Note that since $\mathcal{H}$ is a MMS, the set $R$ is finite.

Let $\operatorname{discr}(\Delta)$ be the points reachable from $\bar{x}$ when using a strategy from $\Sigma_{\Delta}$. All such points are equal to $\bar{x}+\sum_{\vec{r} \in R} i_{\vec{r}} \cdot \Delta \cdot \vec{r}$ for some $i_{\vec{r}} \in \mathbb{N}$. This implies that the set $\operatorname{discr}(\Delta) \cap S$ is finite.

The intuition of our algorithm is the following. Every strategy from $\Sigma_{\Delta}$ can be seen as a function which rather than observing and choosing time delays observes and chooses the number of time periods (multiples of $\Delta$ ) elapsed. Using this abstracted view of strategies, every strategy in $\Sigma_{\Delta}$ corresponds to a strategy in $\Sigma_{\Delta}^{\prime}$ which differs only in the length of the time period. It can be shown that there is a correspondence of points reachable under these two strategies. Seeing the points of $\operatorname{discr}(\Delta)$ as a "grid", the points of $\operatorname{discr}\left(\Delta^{\prime}\right)$ are obtained by stretching (if $\Delta^{\prime}>\Delta$ ) or shrinking (if $\Delta^{\prime}<\Delta$ ) this grid. It follows that for a $\Delta$ to be maximal, there must be a point in $\operatorname{discr}(\Delta)$ which lies on the boundary of $S$, since otherwise the grid $\operatorname{discr}(\Delta)$ can be stretched to some $\operatorname{discr}\left(\Delta^{\prime}\right)$ where $\Delta^{\prime}>\Delta$, preserving the existence of a winning scheduler strategy. Exploiting this property together with the fact that we already know a lower bound on $\Delta_{\max }$ (we can modify line 17 of Algorithm 1 to always schedule modes for $\frac{1}{|\operatorname{vert}(P)|} \cdot \min _{\bar{c} \in \operatorname{vert}(P)} \Delta_{\bar{c}}$ time units), we get only finitely many candidates for maximal $\Delta$, and we can check in each of them whether a winning scheduler strategy exists using Theorem 5.1. Our algorithm is presented as Algorithm 4 .

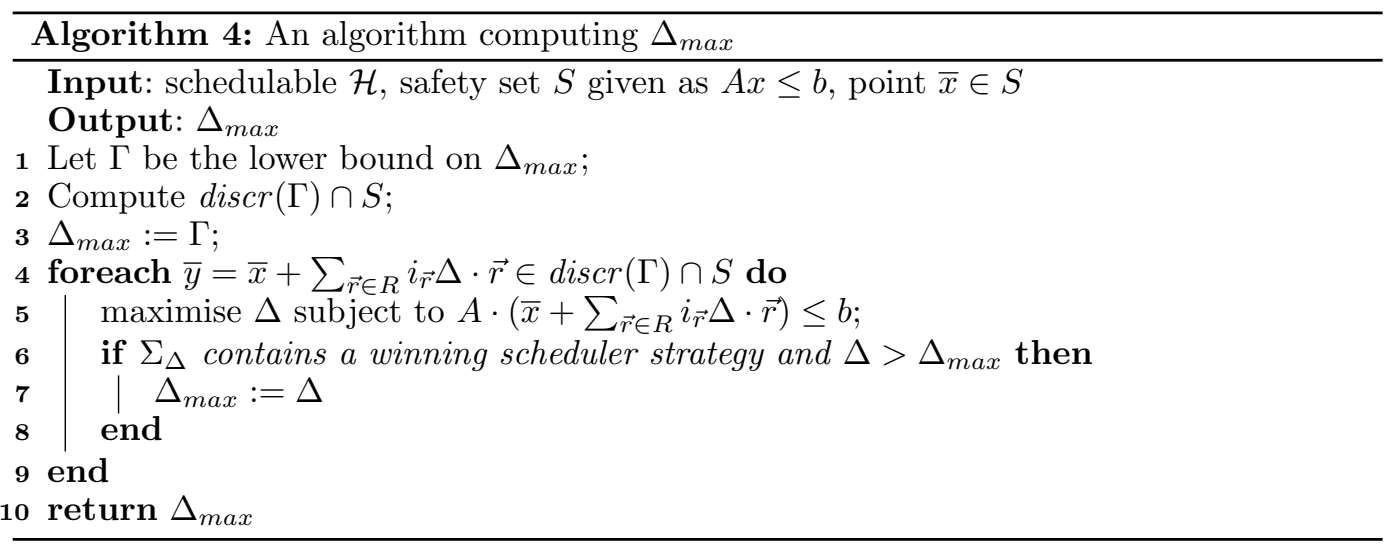

Let us now prove the correctness of the algorithm. Clearly the algorithm terminates in exponential time since the "foreach" loop is executed only exponentially many times at most, and each of the respective lines can be executed in exponential time. Hence, we only need to show that the result returned by the algorithm is correct.

We first introduce some technical notation to capture the intuition of correspondence between points of different discretisations. Define a bijection $g_{\Delta, \Delta^{\prime}}$ between $\operatorname{discr}(\Delta)$ and $\operatorname{discr}\left(\Delta^{\prime}\right)$ that to a point $\bar{x}+\Delta \cdot \sum_{\vec{r} \in R} i_{\vec{r}} \cdot \vec{r}$ where $i_{\vec{r}} \in \mathbb{N}$ assigns the point $\bar{x}+\Delta^{\prime} \cdot \sum_{r \in R} i_{\vec{r}} \cdot \vec{r}$. Intuitively, this function pairs the corresponding points on the "grids" given by $\operatorname{discr}(\Delta)$ and $\operatorname{discr}\left(\Delta^{\prime}\right)$. Note that $g_{\Delta, \Delta^{\prime}}$ is well defined and does not depend on the choice of $i_{\vec{r}} \in \mathbb{N}$ which represent the point and can be non-unique. Indeed, if

$$
\bar{x}+\Delta \cdot \sum_{\vec{r} \in R} i_{\vec{r}} \cdot \vec{r}=\bar{x}+\Delta \cdot \sum_{\vec{r} \in R} i_{\vec{r}}^{\prime} \cdot \vec{r}
$$

for some $i_{\vec{r}}, i_{\vec{r}}^{\prime} \in \mathbb{N}$, then $\sum_{\vec{r} \in R} i_{\vec{r}} \cdot \vec{r}=\sum_{\vec{r} \in R} i_{\vec{r}}^{\prime} \cdot \vec{r}$ and hence also $\bar{x}+\Delta^{\prime} \cdot \sum_{\vec{r} \in R} i_{\vec{r}} \cdot \vec{r}=$ $\bar{x}+\Delta^{\prime} \cdot \sum_{\vec{r} \in R} i_{\vec{r}}^{\prime} \cdot \vec{r}$. 
The following lemma says that when we increase the length of a time period, the set of points on the corresponding grid that are within $S$ can only get smaller.

Lemma 5.3. Let $\Delta \geq \Delta^{\prime}$. Then $g_{\Delta^{\prime}, \Delta}\left(\operatorname{discr}\left(\Delta^{\prime}\right) \cap S\right) \supseteq \operatorname{discr}(\Delta) \cap S$.

Proof. Follows because $S$ is closed, convex and contains $\bar{x}$.

The following lemma intuitively says when we can increase the time period while preserving the existence of a winning scheduler strategy.

Lemma 5.4. Let $\Delta \geq \Delta^{\prime}$ be such that

$$
\operatorname{discr}(\Delta) \cap S=g_{\Delta^{\prime}, \Delta}\left(\operatorname{discr}\left(\Delta^{\prime}\right) \cap S\right)
$$

and assume that there is a winning scheduler strategy in $\Sigma_{\Delta^{\prime}}$. Then there is a winning scheduler strategy in $\Sigma_{\Delta}$.

Proof. Using $g_{\Delta^{\prime}, \Delta}$, we can define a function $h_{\Delta^{\prime}, \Delta}$ from $\Sigma_{\Delta^{\prime}}$ to $\Sigma_{\Delta}$ to capture our intuition of strategies that differ only on the length of a time period as follows. Given $\sigma \in \Sigma_{\Delta^{\prime}}$ and a history

$$
\left\langle\bar{x}_{0},\left(m_{1}, i_{1} \cdot \Delta^{\prime}\right), \vec{r}_{1}, \ldots \bar{x}_{k}\right\rangle
$$

we put

$$
\begin{array}{r}
h_{\Delta^{\prime}, \Delta}(\sigma)\left(\left\langle g_{\Delta^{\prime}, \Delta}\left(\bar{x}_{0}\right),\left(m_{1}, i_{1} \cdot \Delta\right), \vec{r}_{1}, \ldots g_{\Delta^{\prime}, \Delta}\left(\bar{x}_{k}\right)\right\rangle\right) \quad=\sigma\left(\left\langle\bar{x}_{0},\left(m_{1}, i_{1} \cdot \Delta^{\prime}\right), \vec{r}_{1}, \ldots \bar{x}_{k}\right\rangle\right)
\end{array}
$$

Now it is easy to prove by induction that if the set of points that are reachable under $\sigma \in \Sigma_{\Delta^{\prime}}$ is $X$, then the set of points reachable by $h_{\Delta^{\prime}, \Delta}(\sigma) \in \Sigma_{\Delta}$ is equal to $g_{\Delta^{\prime}, \Delta}(X)$.

Now we are ready to proceed with the proof of the correctness of the algorithm. Let $\Delta_{\max }$ be the actual solution (i.e. the supremal number for which the answer to the discrete schedulability problem is Yes), and let $\bar{\Delta}_{\max }$ be the returned number. We know that $\bar{\Delta}_{\max } \leq$ $\Delta_{\max }$, since the algorithm ensures that there is a winning scheduler strategy in $\Sigma_{\bar{\Delta}_{\max }}$. To prove $\bar{\Delta}_{\max } \geq \Delta_{\max }$, it suffices to show that there is a winning scheduler strategy in $\Sigma_{\Delta_{\max }}$, and that $\Delta_{\max }$ is found for some $\bar{y}$ at line 4 of Algorithm 4 .

To show that there is a winning scheduler strategy in $\Sigma_{\Delta_{\max }}$, let

$$
X:=\bigcap_{\Delta<\Delta_{\max }} g_{\Delta, \Delta_{\max }}(\operatorname{discr}(\Delta) \cap S) .
$$

We have $X=\operatorname{discr}\left(\Delta_{\max }\right) \cap S$. The inclusion $\supseteq$ follows by Lemma 5.3 the inclusion $\subseteq$ follows by the fact that $S$ is closed and the fact that as $\Delta$ gets arbitrary close to $\Delta_{\max }$, the points $\bar{y} \in \operatorname{discr}(\Delta)$ get arbitrary close to $g_{\Delta, \Delta_{\max }}(\bar{y})$. By Lemma 5.3 and because $\operatorname{discr}(\Delta) \cap$ $S$ is finite for all $\Gamma \leq \Delta<\Delta_{\max }$, there is $\Delta<\Delta_{\max }$ such that $g_{\Delta, \Delta_{\max }}(\operatorname{discr}(\Delta) \cap S)=X$, and by definition of $\Delta_{\max }$ there is a winning scheduler strategy in $\Sigma_{\Delta}$. Finally by Lemma 5.4 there must be a winning scheduler strategy $\sigma \in \Sigma_{\Delta_{\max }}$.

Now suppose that $\Delta_{\max }$ is not a solution to any of the linear programs executed on line 4 of Algorithm 4 . For each $\bar{y} \in \operatorname{discr}(\Gamma) \cap S$, let $\Delta_{\bar{y}}$ be the solution to the linear program for $\bar{y}$. Let $P$ be the set of all $\bar{y} \in \operatorname{discr}(\Gamma) \cap S$ satisfying $g_{\Gamma, \Delta_{\max }}(\bar{y}) \in \operatorname{discr}\left(\Delta_{\max }\right) \cap S$. Define $\Delta=\min _{\bar{y} \in P} \Delta_{\bar{y}}$. We have $\Delta>\Delta_{\max }$, since if $\Delta=\Delta_{\max }$ then $\Delta_{\max }$ would be the solution to the linear program for the point $\bar{y}$ which realises the minimum, and if $\Delta<\Delta_{\max }$ then $g_{\Gamma, \Delta_{\max }}(\bar{y}) \notin \operatorname{discr}\left(\Delta_{\max }\right) \cap S$. In addition, $g_{\Delta_{\max }, \Delta}\left(\operatorname{discr}\left(\Delta_{\max }\right) \cap S\right)=\operatorname{discr}(\Delta) \cap S$ which by Lemma 5.4 implies that there is a winning scheduler strategy in $\Sigma_{\Delta}$, contradicting the maximality of $\Delta_{\max }$. 


\section{CONCLUSION}

We investigated systems that comprise finitely many real-valued variables whose values evolve linearly based on a rate vector determined by strategies of the scheduler and the environment. We studied an important schedulability problem for these systems, with application to energy scheduling, that asks whether the scheduler can make sure that the values of the variables never leave a given safety set. We showed that when the safety set is a closed convex polytope, existence of non-Zeno winning strategy for the scheduler is decidable for any arbitrary starting state. We also showed how to construct such a winning strategy. On complexity side, we showed that the schedulability problem is co-NP complete in general, but for a fixed number of variables the problem can be solved in polynomial time. Future research includes schedulability problem with respect to more expressive higher-level control objectives including temporal-logic based specification and bounded-rate multi-mode systems with reward functions.

\section{Acknowledgement}

This research was partially supported by NSF award CNS 1035715 and NSF Expeditions in Computing award CCF 1138996. Vojtech Forejt was supported by Newton Fellowship of Royal Society and EPSRC grant EP/M023656/1, and he is also affiliated with Faculty of Informatics, Masaryk University, Brno.

\section{REFERENCES}

R. Alur, V. Forejt, S. Moarref, and A. Trivedi. Safe schedulability of bounded-rate multi-mode systems. In Proceedings of the 16th international conference on Hybrid systems: computation and control, HSCC 2013, April 8-11, 2013, Philadelphia, PA, USA, pages 243-252. ACM, 2013.

R. Alur, A. Trivedi, and D. Wojtczak. Optimal scheduling for constant-rate mulit-mode systems. In $H S C C$, pages 75-84. ACM, 2012.

D. Bhave, S. Jha, S. Narayanan Krishna, S. Schewe, and A.h Trivedi. Bounded-rate multi-mode systems based motion planning. In HSCC, pages 41-50. ACM, 2015.

F. Blanchini. Constrained control for uncertain linear systems. Journal of Optimization Theory and Applications, 71:465-484, 1991.

F. Blanchini. Set invariance in control. Automatica, 35(11):1747-1767, 1999.

Stephen Boyd and Lieven Vandenberghe. Convex Optimization. Cambridge University Press, New York, NY, USA, 2004.

T. A. Henzinger. The theory of hybrid automata. In LICS, pages $278-292$. IEEE Comp. Soc. Press, 1996.

T. A. Henzinger, B. Horowitz, and R. Majumdar. Rectangular hybrid games. In CONCUR 99, pages 320335. Springer, 1999.

T. A. Henzinger and P. W. Kopke. Discrete-time control for rectangular hybrid automata. TCS, 221(12):369-392, 1999.

M. Heymann, L. Feng, G. Meyer, and S. Resmerita. Analysis of Zeno behaviors in a class of hybrid systems. IEEE Tran. on Auto. Ctrl., 50:376-383, 2005.

M. Jurdziński, J. Sproston, and F. Laroussinie. Model checking probabilistic timed automata with one or two clocks. Logical Methods in Comp. Sc., 4(3):12, 2008.

J. Le Ny and G.J. Pappas. Sequential composition of robust controller specifications. In Robotics and Automation, pages 5190-5195, 2012.

J. Liu, N. Ozay, U. Topcu, and R. Murray. Switching protocol synthesis for temporal logic specifications. In American Control Conference, pages 727-734, 2012.

A. Maitra and W. Sudderth. Finitely additive stochastic games with Borel measurable payoffs. Intl. Journal of Game Theory, 27(2):257-267, 1998.

T. X. Nghiem, M. Behl, R. Mangharam, and G. J. Pappas. Green scheduling of control systems for peak demand reduction. In Decision and Control (CDC) and ECC, pages 5131-5136. IEEE, December 2011.

T. X. Nghiem, M. Behl, G. J. Pappas, and R. Mangharam. Green scheduling: Scheduling of control systems for peak power reduction. Intl. Green Computing Conf., pages 1-8, July 2011.

L. T. X. Phan, I. Lee, and O. Sokolsky. Compositional analysis of multi-mode systems. In Euromicro Conf. on Real-Time Sys. (ECRTS), pages 6-9. IEEE, 2010. 